\title{
Optomechanics of Levitated Dielectric Particles
}

\author{
Zhang-qi Yin, ${ }^{1}$ Andrew A. Geraci, ${ }^{2}$ and Tongcang $\mathrm{Li}^{3}$ \\ ${ }^{1}$ The Center for Quantum Information, Institute for Interdisciplinary Information Sciences, \\ Tsinghua University, Beijing 100084, P. R. China \\ yinzhangqi@mail.tsinghua.edu.cn \\ ${ }^{2}$ Department of Physics, University of Nevada, Reno, NV 89557 \\ ageraci@unr.edu \\ ${ }^{3}$ NSF Nanoscale Science and Engineering Center, 3112 Etcheverry Hall, \\ University of California, Berkeley, California 94720, USA \\ tcli@berkeley.edu
}

(Dated: August 22, 2013)

\begin{abstract}
We review recent works on optomechanics of optically trapped microspheres and nanoparticles in vacuum, which provide an ideal system for studying macroscopic quantum mechanics and ultrasensitive force detection. An optically trapped particle in vacuum has an ultrahigh mechanical quality factor as it is well-isolated from the thermal environment. Its oscillation frequency can be tuned in real time by changing the power of the trapping laser. Furthermore, an optically trapped particle in vacuum may rotate freely, a unique property that does not exist in clamped mechanical oscillators. In this review, we will introduce the current status of optical trapping of dielectric particles in air and vacuum, Brownian motion of an optically trapped particle at room temperature, Feedback cooling and cavity cooling of the Brownian motion. We will also discuss about using optically trapped dielectric particles for studying macroscopic quantum mechanics and ultrasensitive force detection. Applications range from creating macroscopic Schrödinger's cat state, testing objective collapse models of quantum wavefunctions, measuring Casimir force, searching short-range non-Newtonian gravity, to detecting gravitational waves.
\end{abstract}

\section{INTRODUCTION}

Classical mechanics is very successful in explaining the motion of macroscopic systems, which are are deterministic, and predictable in principle if initial states of the system are determined. Quantum mechanics, which explains the motion of microscopic systems successfully, on the other hand, is a probabilistic theory. The classical degrees of freedom, such as location and momentum, become wavefunctions in quantum mechanics. While the wavefunction evolves deterministically in quantum mechanics, the wavefunctions can be in superposition states, which is the key difference between quantum and classical physics.

Why macroscopic systems are not in quantum superpostion states? In other words, can we observe Schrödinger's cat states of large objects in laboratory? This question is one of the most outstanding challenges in the modern physics. We may simply explain the quantum-classical world transition by de Broglie wavelength $\lambda$, which is defined as $\lambda=h / p$, where $h$ is Plank constant and $p$ is the momentum. Usually the momentum of macroscopic objects is very large, and the de Broglie wavelength is too small to be observed. Such simple explanation will predict that quantum superpositions of large objects can be observed if we can reduce the momentum $p$ to small enough values, which requires significant cooling. Meanwhile, there are several important models proposed that the quantum-classical transition is due to more profound reasons. For example, Penrose proposed that the conflict between general relativity and quantum mechanics leads to gravity induced collapses of quantum superpositions states [1 $\left[\begin{array}{l}3 \\ ]\end{array}\right.$. Several other intriguing models also proposed that the collapses of massive superposition states might intrinsically be due to quantum mechanics being not complete $[4-6]$. It may be necessary to introduce unknown nonlinear terms to the von Neumann equation to describe large quantum systems [7]. Thanks to the latest experimental developments in macroscopic quantum mechanics [8], some the these models may be tested experimentally soon, which will significantly deepen our understanding of quantum mechanics.

In order to generate and observe the quantum superpostions in macroscopic systems, the momentum (temperature) of the system should be slowed down to the quantum regime. In the past several years, the new research area of optomechanics has had tremendous progress [8, 9]. Quantum ground state cooling of mechanical oscillators by cavity cooling [10, 11] has been realized experimentally 12 14]. For the readers who are interested on the basic theory and development of optomechanics, please read these reviews [8, 9, 15]. Among the implementations of optomechanics, optically levitated dielectric particles have attracted a lot of interest recently 16 25]. As the objects are levitated by optical traps, there is no mechanical contact to the environment, which is the main decoherence source in other mechanical oscillators. Due to the absence of the mechanical contact in this system, the decoherence [26] can be negligible and the oscillation frequency is fully tunable. Thus this system is ideal for study macroscopic quantum mechanics. The center-of-mass (CoM) motion of an optically levitated dielectric particle could be pre-cooled down to 
milli-Kelvin temperatures by feedback [19, 22]. Then, it can be further cooled to the quantum ground state with cavity sideband cooling [16, 17, 23, 27, 28].

After the CoM mode of an optically levitated dielectric particle is cooled down to the quantum regime, macroscopic quantum states, such as quantum superposition states [29, 30], quantum entangled states [16], and squeezed states [16], may be generated. An optically levitated dielectric particle can also be used as an ultra-sensitive detector for Casimir force, non-Newtonian force [31, 32], gravitational waves [33], single molecules collisions [27] and et al.. Besides, the levitated nanoparticles are the best testbed for gravity induced decoherence effects [1, 34], which is the result of the apparent conflict between quantum mechanics and general relativity. Beside CoM motion, the levitated dielectric particle can also rotate freely [35]. The rotation degree of freedom may also be used as an resource for quantum information [36, 37]. The many body physics, such as self-assembly of the nanoparticles in vacuum, was also proposed to study [38, 39].

This review is organized as follows. In Sec. 2, we will review the current status of optical trapping of dielectric particles in air and vacuum. In Sec. 3, we will discuss the CoM motion and cooling of a levitated dielectric particle. In Sec. 4, we will discuss the macroscopic quantum mechanics of levitated dielectric particles. In Sec. 5, we will talk about the applications of a levitated dielectric particle in ultrasensitive force detection.

\section{OPTICAL TRAPPING OF DIELECTRIC PARTICLES IN AIR AND VACUUM}

Optical levitation of dielectric particles in air by an upward-propagating laser beam was first demonstrated by A. Ashkin and J. M. Dziedzic in 1971 [40]. A few

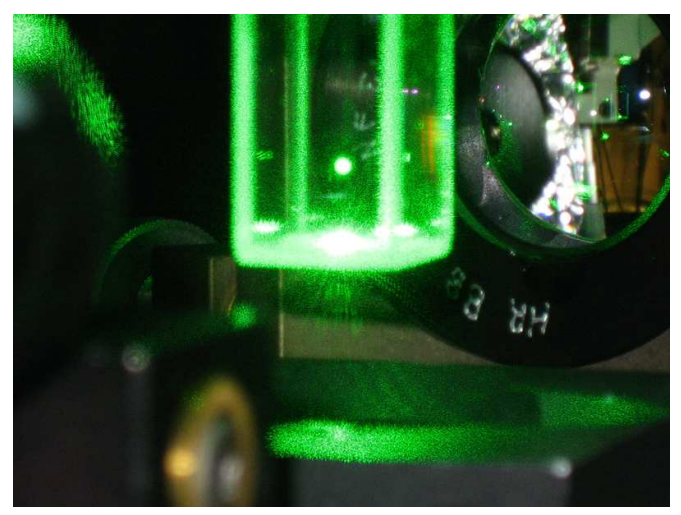

FIG. 1: A 4.7- $\mu \mathrm{m}$ diameter silica microsphere levitated in air inside a glass cell by an upward laser beam. The bright dot near the center of the photo is the trapped microsphere. It appears much larger than the real size of the microsphere because of the overexposure of the camera. Figure adapted from Ref. [42]. years later, optical levitation of microspheres in vacuum at pressures down to $10^{-6}$ torr was achieved [41]. An optical levitation trap is formed by the balance between the scattering force from an upward laser and the gravitational force on a particle. A photo of a $4.7-\mu$ m diameter microsphere levitated by a laser beam in air is displayed in Fig. 1. The trapping frequency of an optical levitation trap is usually very small (about $20 \mathrm{~Hz}$ ) 41], which is too low for quantum ground state cooling.

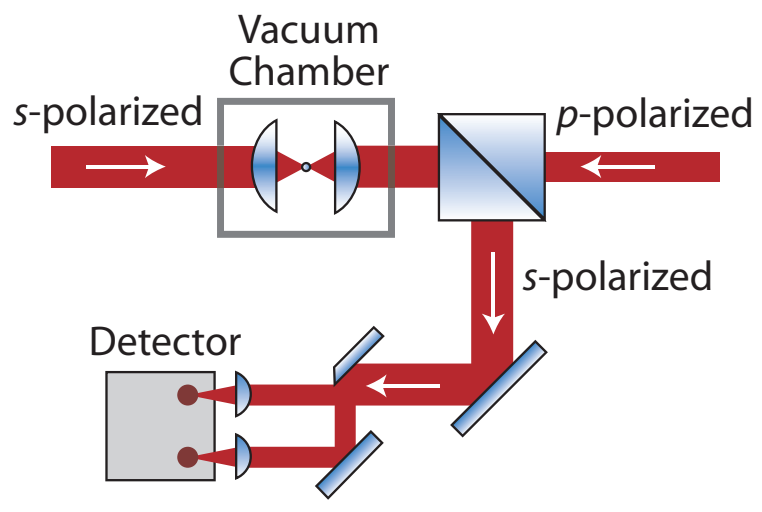

FIG. 2: A counter-propagating dual-beam optical trap in a vacuum chamber. The Brownian motion of a trapped particle will change the direction of the output lasers, which can be used to monitor the instantaneous position of the particle. Figure adapted from Ref. [18].

In 2010, Li et al have trapped glass microspheres in air and high vacuum with a counter-propagating dualbeam trap [18, 19] (Fig. 2). The scattering forces from the two counter-propagating beams cancel, and the gradient force forms a stable three-dimensional (3D) trap. The microspheres were initially stuck on the surface of a glass slide that was placed above the optical trap. They were launched to air by ultrasonic vibration. As they were falling down under the influence of gravity, one of them entered the optical trap and was captured. The trap was very stable and insensitive to the laser power. A 4.7 micrometer particle could be trapped stably when the power of both laser beams were changed from $5 \mathrm{~mW}$ to $2 \mathrm{~W}$. For particles much smaller than the wavelength of the laser, the scattering force is much smaller comparing to the gradient force. Thus nano-particles may be trapped by a single tightly focused laser beam, as was demonstrated by Gieseler et al[22] recently. A mechanical quality factor $(\mathrm{Q})$ of $10^{7}$ has been experimentally demonstrated at $10^{-5} \operatorname{mbar} 22$, and a Q-factor of $10^{8}$ was recently observed at $0.5 \times 10^{-6} \mathrm{mBar}[43$ ]. These values are already higher than the quality factors achieved with clamped oscillators. In ultrahigh vacuum $\left(10^{-10}\right.$ mbar) regime, the quality factor is expected to be higher than $10^{12}$.

Fig. 2 also shows a simple fast detection system that can monitor the trajectory of a trapped particle with ultrahigh resolution [18]. When the trapped particle moves, it will changes the direction of the output laser slightly. 
Thus we can measure the particle position by monitoring the direction of one of the laser that passing through the particle. Li et al have demonstrated a detection sensitivity of about $39 \mathrm{fm} / \sqrt{\mathrm{Hz}}[19]$.

\section{CENTER-OF-MASS MOTION AND COOLING OF A LEVITATED DIELECTRIC PARTICLE}

\section{A. Brownian motion}

An optically trapped microsphere in air (or a nonperfect vacuum) will exhibit Brownian motion due to collisions between the microsphere and air molecules. The Brownian motion was discovered by Robert Brown (1773 - 1858) in 1827 when he used a simple microscope to study the action of particles contained in the grains of pollens. The trajectories of a Brownian particle are commonly thought to be continuous everywhere but not differentiable anywhere, which means the velocity of a Brownian particle is undefined.

In 1907, Einstein published a paper entitled "Theoretical observations on the Brownian motion" in which he considered the instantaneous velocity of a Brownian particle [44]. Einstein showed that by measuring this quantity, one could prove that "the kinetic energy of the motion of the centre of gravity of a particle is independent of the size and nature of the particle and independent of the nature of its environment". This is one of the basic tenets of statistical mechanics, known as the equipartition theorem. However, Einstein concluded that because of the very rapid randomization of the motion, the instantaneous velocity of a Brownian particle would be impossible to measure in practice. In $2010, \mathrm{Li}$ and et al. built a fast detection system with ultrahigh resolution and measured the instantaneous velocity of the Brownian motion of an optically levitated microsphere in air [18].

The distributions of the instantaneous velocities measured by Li et al. are displayed in Fig. 3 They agree with the Maxwell-Boltzmann distribution very well. The measured rms velocities are $v_{r m s}=0.422 \mathrm{~mm} / \mathrm{s}$ at 99.8 $\mathrm{kPa}$ and $v_{r m s}=0.425 \mathrm{~mm} / \mathrm{s}$ at $2.75 \mathrm{kPa}$. These are very close to the prediction of the energy equipartition theorem, $v_{r m s}=\sqrt{k_{B} T / M}$, which is $0.429 \mathrm{~mm} / \mathrm{s}$. As expected, the velocity distribution is independent of pressure. The rms value of the noise signal is $0.021 \mathrm{~mm} / \mathrm{s}$, which means $1.0 \AA$ spatial resolution in $5 \mu$ s. This measurement noise is about $4.8 \%$ of the rms velocity. Fig. 3 represents direct verification of the Maxwell-Boltzmann distribution of velocities and the equipartition theorem of energy for Brownian motion.

The Langevin equation of the Brownian motion of an optically trapped microsphere is:

$$
\frac{d^{2} x_{j}}{d t^{2}}+\Gamma_{0} \frac{d x_{j}}{d t}+\Omega_{j}^{2} x=F_{j}^{t h},
$$

where $\Gamma_{0}$ is the viscous damping factor due to air

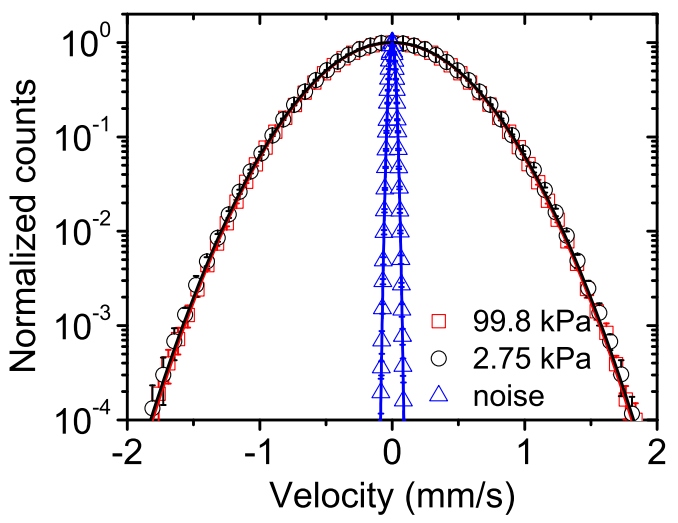

FIG. 3: The distribution of the measured instantaneous velocities of a $3 \mu \mathrm{m}$ silica bead. The statistics at each pressure are calculated from 4 million instantaneous velocities. The solid lines are Maxwell-Boltzmann distributions. Figure adapted from Ref. [18].

molecules, $\Omega_{j} / 2 \pi(\mathrm{j}=1,2,3)$ are the resonant frequencies of the optical trap along the three fundamental axes (x, y, and z axes), and $F_{j}^{t h}=\zeta_{j}(t) \sqrt{2 k_{B} T \Gamma_{0} / M}$ is the Brownian stochastic force. Here $\zeta_{j}(t)$ is the normalized white noise process.

The damping term $\Gamma_{0} \frac{d x}{d t}$ tends to stop any vibration, while the $F_{j}^{t h}$ term drives the motion. It is very interesting that $\Gamma_{0}$ is also contained in $F_{j}^{t h}$ due to fluctuationdissipation theorem. This keeps the average mechanical energy (kinetic and potential energy) of the microsphere to be $k_{B} T$ in each direction at thermal equilibrium.

At thermal equilibrium, the power spectrum of $\mathrm{COM}$ motion of a trapped microsphere along each of the three fundamental mode axes is [45]:

$$
S_{j}(\omega)=\frac{2 k_{B} T_{0}}{M} \frac{\Gamma_{0}}{\left(\Omega_{j}^{2}-\omega^{2}\right)^{2}+\omega^{2} \Gamma_{0}^{2}},
$$

where $\omega / 2 \pi$ is the observation frequency.

\section{B. Feedback cooling}

Since we can measure the instantaneous velocity of the optically trapped dielectric particle, we can cool its $\mathrm{CoM}$ motion by applying a feedback force proportional to the velocity of the particle but with opposite direction (Fig. (4):

$$
F_{j}^{c o o l}=-\Gamma_{j}^{c o o l} \frac{d x_{j}}{d t} .
$$

This force will slow down the motion of the particle. With feedback cooling, the Langevin equation of the Brownian motion of an optically trapped particle is:

$$
\frac{d^{2} x_{j}}{d t^{2}}+\left(\Gamma_{0}+\Gamma_{j}^{c o o l}\right) \frac{d x_{j}}{d t}+\Omega_{j}^{2} x=\zeta_{j}(t) \sqrt{\frac{2 k_{B} T \Gamma_{0}}{M}} .
$$


In contrast to the $\Gamma_{0}$ due to air molecules, $\Gamma_{j}^{c o o l}$ is only contained in the damping term but not in the heating term. Let $\Gamma_{j}^{t o t}=\Gamma_{0}+\Gamma_{j}^{c o o l}$ be the total damping factor, and $T_{j}^{c o o l}=T_{0} \Gamma_{0} / \Gamma_{j}^{t o t}$ be the effective temperature of the motion with feedback cooling, the power spectrum with feedback cooling can be rewritten as:

$$
S_{j}^{c o o l}(\omega)=\frac{2 k_{B} T_{j}^{c o o l}}{M} \frac{\Gamma_{j}^{t o t}}{\left(\Omega_{j}^{2}-\omega^{2}\right)^{2}+\omega^{2}\left(\Gamma_{j}^{t o t}\right)^{2}}
$$

which has the same form as Eq. 2. Because the effective temperature is $T_{j}^{c o o l}=T_{0} \Gamma_{0} /\left(\Gamma_{0}+\Gamma_{j}^{c o o l}\right)$, the motion can be cooled significantly by applying a feedback damping $\Gamma_{j}^{c o o l}>>\Gamma_{0}$.

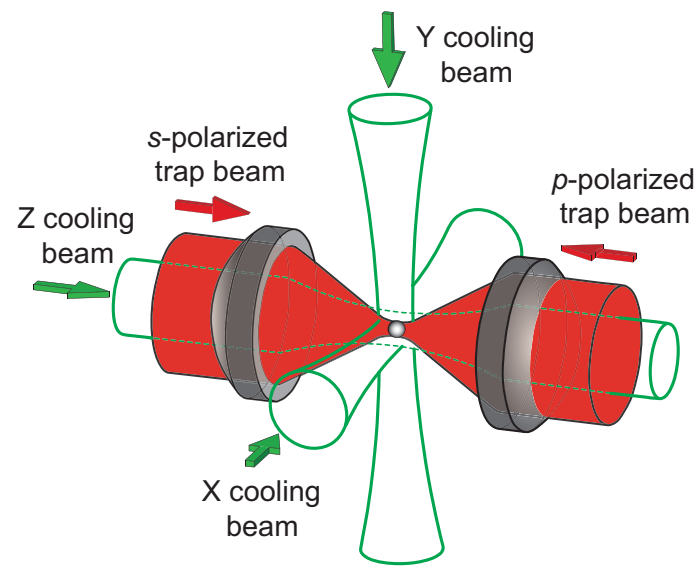

FIG. 4: Simplified schematic showing a glass microsphere trapped at the focus of a counter-propagating dual-beam optical tweezer $(1064 \mathrm{~nm})$, and three $532 \mathrm{~nm}$ laser beams along the axes for cooling. Figure adapted from Ref. [19].

Figure 5 show experimental results of feedback cooling along $\mathrm{Y}$ axis by Li et al [19]. Before feedback is turned on, the resonant frequencies $\left(\omega_{j} / 2 \pi\right)$ are $8066 \pm 5 \mathrm{~Hz}$, $9095 \pm 4 \mathrm{~Hz}$, and $2072 \pm 6 \mathrm{~Hz}$ for the fundamental modes at $637 \mathrm{~Pa}$ along the $\mathrm{X}, \mathrm{Y}$, and $\mathrm{Z}$ axes, respectively. After the feedback cooling circuits were turned on, the temperature of the $\mathrm{Y}$ mode changed from $297 \mathrm{~K}$ to $24 \mathrm{~K}$ at $637 \mathrm{~Pa}$. The mode temperature is obtained by fitting the measured power spectrum with Eq. 5. Then Li et al reduced the air pressure while keeping the feedback gain almost constant, thus the heating rate due to collisions from air molecules decreases, while the cooling rate remains constant. As a result, the temperature of the motion dropped. At $5.2 \mathrm{mPa}$, the mode temperatures were $150 \pm 8 \mathrm{mK}, 1.5 \pm 0.2 \mathrm{mK}$, and $68 \pm 5 \mathrm{mK}$ for the $\mathrm{x}$, $\mathrm{y}$ and $\mathrm{z}$ modes. The mean thermal occupation number $\langle n\rangle=k_{B} T_{j}^{f b} / \hbar \omega_{j}$ of the $\mathrm{y}$ mode is reduced from about $6.8 \times 10^{8}$ at $297 \mathrm{~K}$ to about 3400 at $1.5 \mathrm{mK}$.

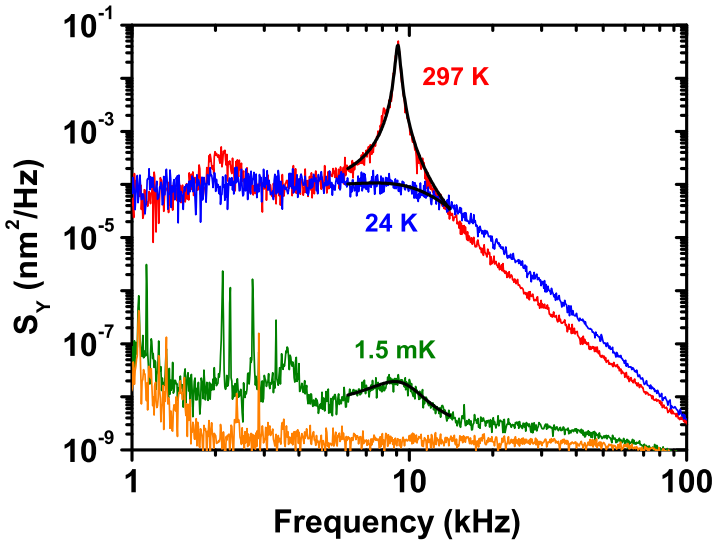

FIG. 5: Power spectra of a trapped $3-\mu \mathrm{m}$ diameter microsphere along the $\mathrm{Y}$ axis as it is cooled. The red curve is the intrinsic spectrum at $637 \mathrm{~Pa}$ without feedback cooling, the blue curve is the spectrum at $637 \mathrm{~Pa}$ with feedback cooling, the green curve is the spectrum at $5.2 \mathrm{mPa}$ with feedback cooling, and the orange curve is the noise signal when there is no particle in the optical trap. The black curve is the fit of a thermal model (see text for details). The mode temperatures are obtained from these fits. Figure adapted from Ref. [19].

\section{Cavity sideband cooling}

In 2009, two groups proposed to use the cavity sideband cooling scheme [10, 11] to cool the CoM mode of optically levitated nanoparticle down to the ground state 16, 17]. Recently, the cavity cooling was partially realized by Kiesel et al. 23] and Asenbaum et al. [24]. Kiesel et al. have optically trapped a nanoparticle inside the optical cavity, and achieved the sideband limit 23. Because of the relatively high pressure $(4 \mathrm{mbar})$ in their experiment, they were only able to cool the effective temperature of a levitated nanoparticle from room temperature to about $64 \mathrm{~K}$. They believe that the quantum ground state may approach, if they can increase the vacuum to $10^{-7}$ mbar. Here, we will give a short review on the theory of cavity sideband cooling of optically trapped nanoparticle.

The typical scheme of cavity sideband cooling for optically trapped nanoparticle is show in Fig. 6. The optical trap is placed in the optical cavity, and a nanosphere with mass $m$ is loaded in the trap. We denote vibration frequency of the nanoparticle along the $z$ axis be $\omega_{m}$, the frequency of the cooling laser as $\omega_{l}$, the resonant frequency of the cavity as $\omega_{c}$, the intrinsic cavity linewidth as $\kappa$ and the rate of a photon scattered by the microsphere as $\gamma_{s c}$. The sideband cooling requires that the linewidth $\kappa$ is much shorter than the optical trap frequency $\omega_{m}$. As the size of nanoparticle is much less than the wavelength of the trapping and cooling light, and the molecule collision rate is very low at high vaccum, the photon scattering decoherence rate $\gamma_{r c}$ is usually much less than $\kappa$, and can be neglected. 


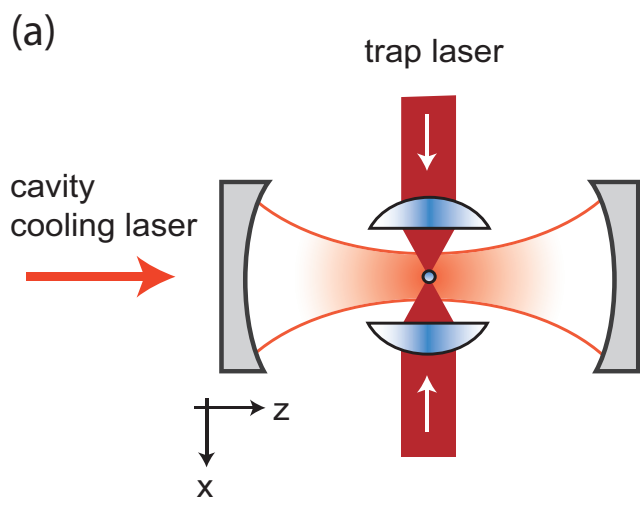

(b)

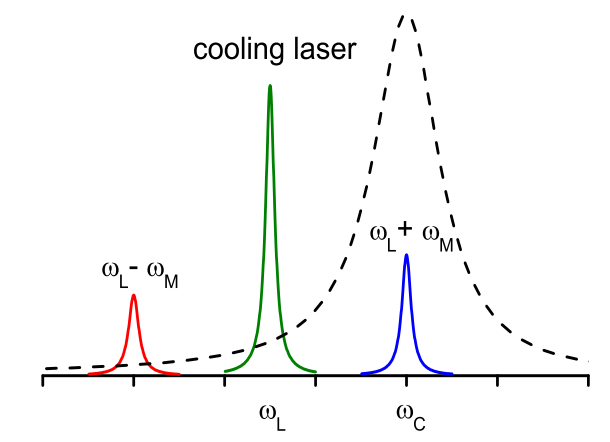

FIG. 6: (a) Scheme of 1D cavity cooling. A nanoparticle is trapped inside an optical cavity with a dual-beam trap. (b) Principle of 1D cavity cooling. The frequency of the cooling laser $\omega_{l}$ is slightly smaller than the resonant frequency of the optical cavity $\omega_{c}$. The mechanical vibration of the trapped microsphere at frequency $\omega_{m}$ induces two side bands of the laser at frequencies of $\omega_{l}+\omega_{m}$ and $\omega_{l}-\omega_{m}$.

Let us consider a nanoparticle at position $z$ moving with momentum $p$ along the $z$ axis inside of a driven cavity. The nanoparticle causes the cavity frequency to shift by an amount

$$
\delta \omega_{c}=-\frac{1}{2} \frac{\int d^{3} \mathbf{r} \delta P(\mathbf{r}) \dot{\mathbf{E}}(\mathbf{r})}{\int d^{3} \mathbf{r} \epsilon_{0} \mathbf{E}^{2}(\mathbf{r})} \cdot \omega_{c 0},
$$

where $\omega_{c 0}$ is the resonant frequency of a cavity without the nanosphere, $\mathbf{E}(\mathbf{r})$ is the cavity mode profile and $\delta P(\mathbf{r})$ is the variation in permittivity induced by the nanosphere. Due to the tiny scale of the nanosphere (much less than laser wavelength), we can use can use Rayleigh approximation, and have $P\left(\mathbf{r}^{\prime}\right) \simeq \alpha_{\text {ind }} E(\mathbf{r}) \delta(\mathbf{r}-$ $\mathbf{r}^{\prime}$ ), with $\mathbf{r}$ the CoM position of the nanosphere, $\alpha_{\text {ind }}=$ $3 \epsilon_{0} V\left(\frac{\epsilon-1}{\epsilon+2}\right)$ the polarizability, $V$ the sphere volume, and $\epsilon$ is the electric permittivity.

The Hamiltonian of the system can be approximated as

$H_{e f f}=\hbar \omega_{m} a_{m}^{\dagger} a_{m}-\hbar \Delta_{c} a_{c}^{\dagger} a_{c}+\frac{\hbar \Omega_{c}}{2}\left(a_{c}+a_{c}^{\dagger}\right)+\hbar g_{j} a_{c}^{\dagger} a_{c}\left(a_{m}+a_{m}^{\dagger}\right.$ where $g_{j}=q_{\text {zpf } j} \partial U(z) /\left.\partial j\right|_{z=z_{0}}$ characterizes the coupling strength between the cavity mode and the oscillation of the nanosphere, $U(z)$ is the nanoparticle induced frequency shift, and $z_{\mathrm{zpf}}=\sqrt{\hbar / 2 m \omega_{j}}$ is zero-point fluctuation for the phonon mode $a_{m} . \Delta_{c}=\omega_{c}-\omega_{l}$ is detunings between the lasers and the cavity modes $a_{c} . \Omega$ is the driving strength of the cooling laser.

From Eq. (6), the linearized Langevin equations of motion for our system are,

$$
\begin{aligned}
\dot{a_{c}} & =\left(i \Delta_{c}^{\prime}-\kappa_{j} / 2\right) a_{c}-i g \alpha_{c}\left(a_{m}+a_{m}^{\dagger}\right)+\sqrt{\kappa_{j}} a_{c}^{\text {in }}, \\
a_{m} & =-i \omega_{m} a_{m}-i g\left(\alpha_{c} a_{c}^{\dagger}+\alpha_{m}^{*} a_{c}\right),
\end{aligned}
$$

where $\alpha_{c}=i \Omega /\left(2 i \Delta_{c}^{\prime}-\kappa\right), \Delta_{c}^{\prime}=\Delta_{c}+2 g_{j}^{2}\left|\alpha_{c}\right|^{2} / \omega_{m}, \alpha_{c}$ is the amplitude of cavity mode $a_{c}$, and $\Delta_{c}^{\prime}$ is the effective detuning between the driving laser and the cavity mode $a_{c}$. The linearization of the Langevin equations is valid only if the state is stable. The stable criteria is [27] $S_{1}=4 \Delta_{c}^{\prime} \omega_{m} g^{2} \alpha_{c}^{2} \kappa^{2}>0, S_{2}=\omega_{m} \Delta_{c}{ }^{2}-g^{2} \alpha_{c}^{2} \Delta_{c}^{\prime}>0$. Because of $\Delta_{c}^{\prime}>0$, the criteria $S_{1}$ is always valid. The criteria $S_{2}$ are valid only when $g \alpha_{c}<\sqrt{\omega_{m} \Delta_{c}^{\prime}}$. To realize resolved sideband cooling, we require $\omega_{m} \gg \kappa$. We suppose $\left|g \alpha_{c}\right| \ll \kappa$, and find that the final phonon number is

$$
n_{m}=-\frac{\left(\omega_{m}+\Delta_{c}^{\prime}\right)^{2}+(\kappa / 2)^{2}}{4 \omega_{m} \Delta_{c}^{\prime}}
$$

In the special case of $\Delta_{c}^{\prime}=-\omega_{m}$, the final phonon number is $n_{m}=\left(\kappa / 4 \omega_{m}\right)^{2} \ll 1$. The cooling rate is $\Gamma=g^{2}\left|\alpha_{c}\right|^{2} /\left[\kappa\left(1+\frac{\kappa^{2}}{16 \omega_{m}^{2}}\right)\right]$.

\section{D. $3 \mathrm{D}$ sideband cooling}

A nanoparticle will scatter the trapping/cooling laser to all three dimensions and cause 3D heating. In order to achieve ground state cooling of an optically trapped nanosphere, we must use a 3D cooling scheme. We can add two more cavities for cooling the other two dimensions, but the system will become too complex to be realized experimentally. A better method to cool and measure the 3D motion of a nanosphere is to use the TEM00, TEM01, and TEM10 modes of a single cavity, as proposed by Yin et al. 27]. The TEM01 and TEM10 beams can be generated from a TEM00 beam by two phase plates. Each one of these three modes can be coupled to the motion of a trapped nanosphere along one orthogonal axis.

As shown in Fig. 7, we consider an optically trapped nanosphere with mass $m$ confined in a cavity by means of an optical tweezer [18]. The frequencies of the optical trap along the $z, x$, and $y$ axes are $\left(\omega_{1}, \omega_{2}\right.$, and $\left.\omega_{3}\right)$. Beside the conventional method of using a cooling laser with TEM00 mode to cool the motion along $z$ direction, we add two non-Gaussian beams with TEM01 and TEM10 modes to drive the cavity in order to cool the motion along the $x$ and $y$ directions, respectively. 


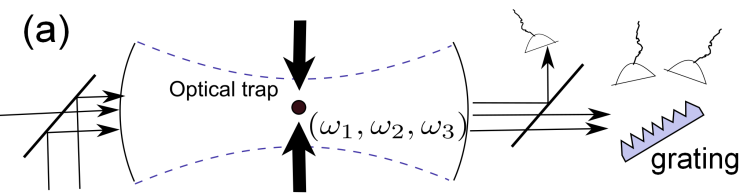

(b)

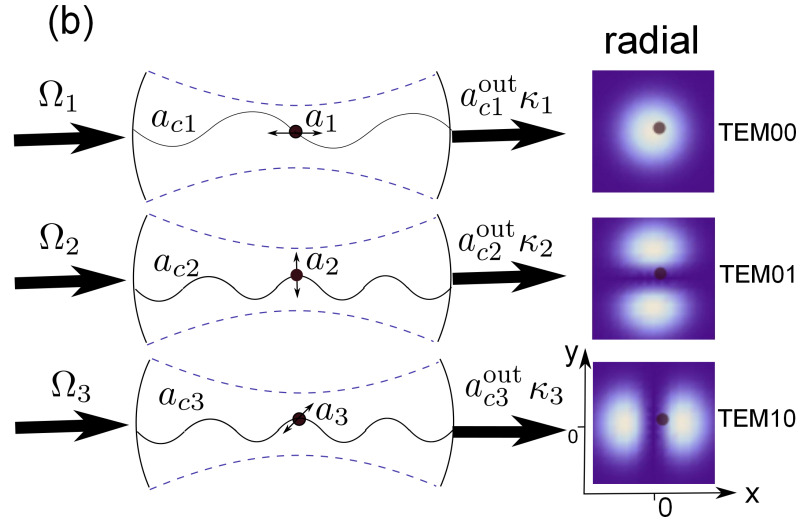

FIG. 7: (a) Cooling and detecting scheme. A nanosphere is trapped by a dual-beam optical tweezer inside of a cavity. The cavity is driven by three lasers in TEM00, TEM01 and TEM10 modes. The TEM01 mode laser has different polarization, and is separated from the other two lasers by a polarizing beam splitter for detection. The TEM00 and TEM01 lasers have different frequencies, and are separated by a grating for detection. (b) Three cooling modes TEM00, TEM01, and TEM10, and their radial distribution. The black dot represents the position of a trapped nanosphere. Figure adapted from Ref. [27]

The resonant frequencies of the cavity modes $a_{c 1}, a_{c 2}$, and $a_{c 3}$ are $\omega_{c 1}, \omega_{c 2}$, and $\omega_{c 3}$, respectively. The detunings between the lasers and the cavity modes are $\Delta_{c j}=\omega_{c}^{j}-\omega_{L}^{j}(j=1,2,3)$. We suppose that the TEM01 and TEM10 lasers have the same frequency, but with orthogonal polarization. The TEM00 and TEM01 lasers have the same polarization, but different frequencies. In practical, the frequency differences between TEM00 and TEM01 (TEM10) could be very large, and the TEM01 and TEM10 modes are orthogonal in polarizations. Therefore the interference between the three cavity modes can be neglected.

The total Hamiltonian of the system in the rotating frame is 27.

$$
H=\sum_{j=1}^{3}\left[\hbar \omega_{j} a_{j}^{\dagger} a_{j}-\hbar\left(\Delta_{j}-U_{j}\right) a_{c j}^{\dagger} a_{c j}+\frac{\hbar \Omega_{j}}{2}\left(a_{c j}+a_{c j}^{\dagger}\right)\right],
$$

where $a_{j}$ characterizes the phonon mode along $q_{j}$ direction with $q_{1}=z, q_{2}=x, q_{3}=y . \quad \Omega_{j}$ is the driving strength by the lasers and $U_{j}$ characterizes the coupling between the cavity mode $a_{c j}$ and the nanosphere. In the limit that $\epsilon \gg 1$, where $\epsilon$ is the electric permittivity of the nanosphere, we get [16]

$$
\begin{aligned}
& U_{1}=-\frac{3 V}{2 V_{c 1}} \exp \left(-\frac{2 x^{2}+2 y^{2}}{w^{2}}\right) \cos ^{2}\left(k_{1} z+\varphi_{1}\right) \omega_{c 1}, \\
& U_{2}=-\frac{3 V}{2 V_{c 2}} \frac{x^{2}}{w^{2}} \exp \left(-\frac{2 x^{2}+2 y^{2}}{w^{2}}\right) \cos ^{2}\left(k_{2} z+\varphi_{2}\right) \omega_{c 2}, \\
& U_{3}=-\frac{3 V}{2 V_{c 3}} \frac{y^{2}}{w^{2}} \exp \left(-\frac{2 x^{2}+2 y^{2}}{w^{2}}\right) \cos ^{2}\left(k_{3} z+\varphi_{3}\right) \omega_{c 3},
\end{aligned}
$$

with $V_{c 1}=(\pi / 4) L w^{2}$ and $V_{c 2}=V_{c 3}=(\pi / 16) L w^{2}$.

We assume the optical tweezer to be much stronger than the cavity-mode-induced trap, and neglect the effects of cooling lights on trapping. Besides, if we carefully choose the location of the trap, such as $z_{0}=0$, $x_{0}=y_{0}=0.25 \mathrm{w}, \varphi_{1}=\pi / 4$, and $\varphi_{2}=\varphi_{3}=0$, the gradients of the three light fields lie approximately along the three axes. The effective Hamiltonian is

$$
\begin{aligned}
H_{e f f}= & \sum_{j=1}^{3}\left[\hbar \omega_{j} a_{j}^{\dagger} a_{j}-\hbar \Delta_{j} a_{c j}^{\dagger} a_{c j}+\frac{\hbar \Omega_{j}}{2}\left(a_{c j}+a_{c j}^{\dagger}\right)\right. \\
& \left.+\hbar g_{j} a_{c j}^{\dagger} a_{c j}\left(a_{j}+a_{j}^{\dagger}\right)\right],
\end{aligned}
$$

where $g_{j}=q_{\mathrm{zpf} j} \partial U(x, y, z) /\left.\partial j\right|_{x=x_{0}, y=y_{0}, z=z_{0}}$ characterizes the coupling strength between the cavity mode and the oscillation of the nanosphere, and $q_{\mathrm{zpf} j}=\sqrt{\hbar / 2 m \omega_{j}}$ is zero-point fluctuation for the phonon mode $a_{j}$. In general, $g_{1}$ can be one to two orders larger than $g_{2}$ and $g_{3}$.

As the 3D motional modes of the system are decoupled with each other in effective Hamiltonian (9), we can find the final phonon number equation with the similar method discussed in the previous subsection [IIC $n_{m j}=$ $-\frac{\left(\omega_{j}+\Delta_{c j}^{\prime}\right)^{2}+\left(\kappa_{j} / 2\right)^{2}}{4 \omega_{j} \Delta_{c j}^{\prime}}$. In the special case of $\Delta_{c j}=-\omega_{j}^{\prime}$, the final phonon number is $n_{m j}=\left(\kappa_{j} / 4 \omega_{j}\right)^{2} \ll 1$.

\section{E. Noise and decoherence}

Here we briefly discuss the noise and decoherence in optically levitated nanoparticles system. The dominant noise sources for the CoM mode of nanoparticles are collisions with a background gas and momentum recoil kicks due to scattered photons. The noise contributions from shot noise, blackbody radiation are negligible [16]. For collisions with a background gas, it is found that the the damping rate of the phonon is $\gamma_{g}=(16 / \pi)(P /$ vr $\rho)$, where $P$ and $v$ are background gass pressure and mean speed, $r$ is the radius of the sphere, and $\rho$ is the density of the nanosphere. For $\omega_{m}=0.5 \mathrm{MHz}, r=50$ nm, room temperature gas with $P=10^{-10}$ Torr, we find that $\gamma_{g}=10^{-6} \mathrm{~s}^{-1}$. Therefore, the molecules collision induced decoherence is also very small. In fact, we can directly measure this collision by output mode [27]. We will discuss this in the next section. Photon scattering will entangle the mechanical mode and output light, and leads to heating of the mechanical mode, too. Considering motion only along the $\mathrm{z}$ direction, it is found 
that [16] $\gamma_{s c}=(2 / 5)\left(\omega_{r} / \omega_{m}\right) R_{s c}$, where $\omega_{r}=\hbar k^{2} / 2 \rho V$ is the recoil frequency, $R_{s c}=24 \pi^{3} \frac{I_{0}}{\lambda^{4}} \frac{V^{2}}{\hbar \omega_{c}}\left(\frac{\epsilon-1}{\epsilon+2}\right)^{2}$ is the photon scattering rate for sphere. The photon scattering rate could be very large $\left(R_{s c} \sim 10^{14} \mathrm{~s}^{-1}\right.$ for $I_{0}=1 \mathrm{~W} / \mu \mathrm{m}^{2}$ and $r=50 \mathrm{~nm}$ ), while the momentum of photons is much smaller than that of background air molecules. It is convenient to define a dimensionless parameter $\phi=\frac{\gamma_{s c}}{\omega_{m}}=\frac{4 \pi^{2}}{5} \frac{\epsilon-1}{\epsilon+2}\left(V / \lambda^{3}\right)$ [16], which can be much less than 1 if $V$ of nanophere is much less than $\lambda^{3}$. Therefore, if we want to decrease the photon recoil heating, we should trap smaller nanoparticle, or use trapping and cooling laser with longer wavelength. We may also use magnetic force to trap the nanoparticle, where the photon scattering effect is negligible [46, 47].

Then we consider the heating effects from the optical trap [48]. The heating mainly comes from the laser intensity fluctuation and the laser-beam-pointing noise. For the former, we define the fluctuations of the laser $\epsilon(t)=\left(I(t)-I_{0}\right) / I_{0}$, with $I_{0}$ the average intensity and $I(t)$ the laser intensity at time $t$. By using first-order time-dependent perturbation theory, we get $\langle\dot{E}\rangle=\frac{\pi}{2} \omega_{j}^{2} S_{\epsilon}\left(2 \omega_{j}\right)\langle E\rangle$ [48]. The heating constant is $\Gamma_{\epsilon}=$ $\frac{\pi}{2} \omega_{j}^{2} S_{\epsilon}\left(2 \omega_{j}\right)$, where $S_{\epsilon}(\omega)=\frac{2}{\pi} \int_{0}^{\infty} d \tau \cos (\omega \tau)\langle\epsilon(t) \epsilon(t+\tau)\rangle$ is the one-sided power spectrum of the fractional intensity noise, which could be on the order of $10^{-14} \mathrm{~Hz}^{-1}$. For the trap frequency of $\mathrm{MHz}, \Gamma_{\epsilon}$ approaches the order of $10^{-1} \mathrm{~Hz}$. The laser-beam-pointing noise is originated from the fluctuation relevant to the location of the trap center, which is independent of the phonon energy. Similarly, we may get $\langle\dot{E}\rangle=\frac{\pi}{2} m \omega_{j}^{4} S_{j}\left(\omega_{j}\right)$, where $j=x, y, z$, and $S_{j}(\omega)$ is the noise spectrum of location fluctuations. We define the heating rate as $\Gamma_{j}=\frac{\pi}{2} m \omega_{j}^{4} S_{j}\left(\omega_{j}\right) /\left(\hbar \omega_{j}\right)$, which represents phonon number increase per second. If we set $\Gamma_{j}$ to be on the order of $10^{-1} \mathrm{~Hz}$, we should make sure that $S_{j}\left(\omega_{j}\right)$ is around $10^{-35} \mathrm{~m}^{2} / \mathrm{Hz}$ for $\omega_{j} \sim$ $1 \mathrm{MHz}$. Experimentally $S_{j}(\omega)$ has been controlled less than $10^{-34} \mathrm{~m}^{2} / \mathrm{Hz}$ for $\omega \sim 2 \pi \mathrm{kHz}$ [49]. With the increase of the optical trap frequency to large detuning from the system's resonant frequency, $S_{j}\left(\omega_{j}\right)$ is dropping down quickly. Therefore, we believe that the laser-beampointing noise could be well controlled and the heating rate $\Gamma_{j}$ would be less than $0.1 \mathrm{~Hz}$.

The phase noise induced by the cooling laser also need to be seriously considered [26, 50, 51]. Because the cooling laser is of finite linewidth, the laser field can be wrote down as $\varepsilon(t)=\varepsilon e^{i \phi(t)}$. We assume the phase noise $\phi(t)$ to be Gaussian and with zero mean value. For the Lorentzian noise spectrum with $S_{\dot{\phi}}(\omega)=$ $2 \Gamma_{L} \gamma_{c} /\left(\gamma_{c}^{2}+\omega^{2}\right)$, and correlation function $\left.\{\dot{\phi} \dot{s}) \phi\left(\dot{s}^{\prime}\right)\right\}=$ $\Gamma_{L} \gamma_{c} \exp \left(-\gamma_{c}\left|s-s^{\prime}\right|\right)$, where $\Gamma_{L}$ is the linewidth of the laser and $\gamma_{c}^{-1}$ is the correlation time of the laser phase noise, the phonon number limited by this noise is $n_{p h}>$ $n_{c} \frac{\Gamma_{L}}{\kappa} \frac{\gamma_{c}^{2}}{\gamma_{c}^{2}+\omega_{j}^{2}}$ [51]. If we choose $\Gamma_{L}=1 \mathrm{kHz}, \gamma_{c}=3 \mathrm{kHz}$, $\omega_{j}=10^{6} \mathrm{~Hz}$, and $n_{c}=10^{7}$, we have $n_{p h} \ll 1$. Besides, we may use double resonance scheme to further increase the cooling rate and suppress the phase noise [26, 52].

\section{MACROSCOPIC QUANTUM MECHANICS}

After the CoM mode of optically trapped nano(micro)particle being cooled to the quantum ground state, a lot of quantum states can be generated, and many interesting quantum phenomena could be observed in this macroscopic systems. In this section, we will summarize the recent developments in this direction.

\section{A. State transfer and applications}

In the previous section, we focused on the CoM mode of the nanoparticle and calculated the steady phonon number when the cooling laser is on. In fact, the cooling laser also realizes the quantum interface between cavity mode and the phonon mode. With the interface, we can archive quantum state transfer between the cavity and phonon modes [16]. From Eq.(7), we have a reduced equation under rotating wave approximation, in the case of $\Delta_{c j}^{\prime}=-\omega_{m}$ and $\omega_{m} \gg \kappa, \alpha_{c} g$, as [27],

$$
\begin{aligned}
\dot{a_{c}} & =-\frac{\kappa}{2} a_{c}-i g \alpha_{c} a_{m}+\sqrt{\kappa} a_{c}^{\text {in }}, \\
a_{m} & =-i g \alpha_{c} a_{c} .
\end{aligned}
$$

In the limit $\kappa \gg g \alpha_{c}$, using boundary condition $a_{c}^{\text {out }}=$ $-a_{c}^{\text {in }}+\sqrt{\kappa} a_{c}$, we get $a_{c}^{\text {out }}=-i \frac{2 g \alpha_{c}}{\sqrt{\kappa}} a_{m}+a_{c}^{\text {in }}, \dot{a}_{m}=$ $-\frac{2 g^{2} \alpha_{c}^{2}}{\kappa} a_{m}-\frac{2 i g \alpha_{c}}{\sqrt{\kappa}} a_{c}^{\text {in }}$. Therefore the CoM motion of the nanosphere can be mapped to the cavity output fields. Physically, the cooling mechanism can be viewed as transferring phonon excitation to the cavity mode, and finally leaking out of cavity.

The quantum state transfer between photon and phonon modes has many applications. The first one is generating superposition state $(|0\rangle+|1\rangle) / \sqrt{2}$ [17], where $|0\rangle(|1\rangle)$ is the ground state (first Fock state) of the CoM phonon mode. We impinge a single-photon state into the cavity. Part of the photon will reflect, and part of it transmit. In present of cooling laser, the Langevin equations (10) swap the photon state into the CoM phonon state of nanoparticle. We the entangle state $|0\rangle_{r}|1\rangle_{m}+|1\rangle_{r}|0\rangle_{m}$, where $r$ denotes the reflecting photon mode, and $m$ denotes the CoM phonon mode. The motional state collapses into the superposition state $\Psi\rangle=c_{0}|0\rangle_{m}+c_{1}|1\rangle_{1}$, by performing a balanced homodyne measurement and by switching off the driving field. Here the coefficients $c_{0(1)}$ depend on the measurement result. This state can be detected by transferring it back to the cavity with a red-detuning laser and then performing tomography on the output field.

The second application of the quantum state transfer is generating the squeezed state of light [16]. In order to generate the squeezed light we need to creat the mechanical squeezed state, then transfer the squeezed properties to the output light by quantum state transfer. We add a sinusoidally varying component to the intensity of the 
trapping beam, which yields the Hamiltonian of a parametric amplifier

$$
H_{s}=\epsilon_{m} \omega_{m}^{2} z^{2} \sin 2 \omega_{m} t
$$

Here $\epsilon_{m}$ is a small parameter characterizing the strength of the modulation of the trap frequency. We are interested in the outgoing light over a narrow frequency range near the cavity resonance, specifically considering $\mathbf{X}_{ \pm \text {,out }}(\omega=0)$. Taking the limit as one approaches threshold and $\Gamma=\kappa$, the variance in the output light is given by [16]

$$
\Delta \mathbf{X}_{+, \text {out }}^{2}(\omega=0)=\frac{5}{16} \frac{\kappa^{2}}{\omega_{m}^{2}}
$$

Here we neglect the phonon recoil heating effects. We find that the output squeezing could easily approach to $30 \mathrm{~dB}$ of noise reduction relative to the vacuum state.

We can use quantum state transfer to realize entanglement transfer between remote nanoparticles trapped in separate cavities [16], or to generate the Schrödingers cat state $|\alpha\rangle+|-\alpha\rangle$ [17]. Besides, if we drive the light on resonance of blue sideband, we will generate two-mode squeezed state between phonon and photon modes. By combing blue and red sideband driving, we can generate two-mode squeezed light with the method similar as the Ref. 53]. However, as there is no non-linear coupling in CoM mode of trapped nano-particles (at least for the first order), the non-Gaussian state of mechanical mode can only be generated by mapping the photon state into it. Therefore, the quantum state that can be generated(detected) in trapped nanoparticle is depending on the input(output) state of light, which highly limits the applications of the system.

\section{B. Optically trapped nanoparticle with built-in spins}

Nanodiamonds with NV centers have been recently trapped by optical tweezers in fluid [55, 56] and atmospheric air [57], and similar technologies can be used to optically trap them in vacuum [22]. The nonlinear interaction required for generation of non-Gaussian quantum states is enabled through the spin-mechanical coupling with a built-in nitrogen-vacancy center inside the nanodiamond [54]. By detecting the spin state of NV center in nanocrystal diamond, the phonon state can be detected without cavity mode.

As shown in Fig. 8, we consider a nanodiamond of mass $m$ optically trapped in vacuum with trapping frequency $\omega_{m}$. The motion of its CoM mode $a_{m}$ is described by the Hamiltonian $H_{m}=\hbar \omega_{m} a_{m}^{\dagger} a_{m}$. The nanodiamond has a built-in NV center with its level configuration shown in Fig.1b in the ground-state manifold. The NV spin is described by the Hamiltonian $H_{\mathrm{NV}}=\hbar\left(\omega_{+1}|+1\rangle\left\langle+1\left|+\omega_{-1}\right|-1\right\rangle\langle-1|\right)$, where we have set $|0\rangle$ as the energy zero point. A magnet tip near (a)

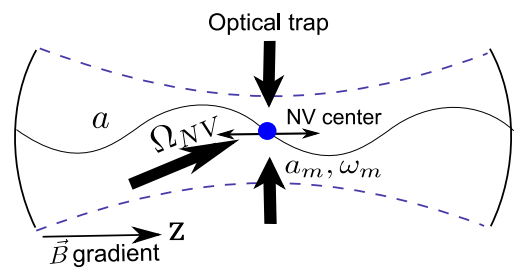

(b)

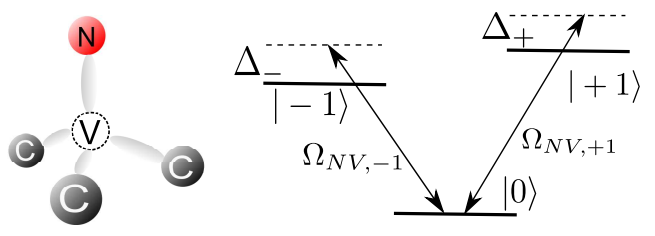

FIG. 8: (a) A nanodiamond with a NV center is optically trapped in vacuum with spin-mechanical coupling enabled through a nearby magnetic tip and opto-mechanical coupling through a cavity around. (b) The atomic structure (left) and the level diagram (right) in the ground state manifold for a $\mathrm{NV}$ center in the nanodiamond. Figure adapted from Ref. [54].

the NV center induces a strong magnetic field gradient [58], which couples the electron spin and the CoM oscillation of the nanodiamond. The coupling Hamiltonian is denoted by $H_{\mathrm{NV} m}=\hbar \lambda S_{z}\left(a_{m}+a_{m}^{\dagger}\right)$ [59, 60], where $S_{z} \equiv|+1\rangle\langle+1|-|-1\rangle\langle-1|$. The coupling strength $\lambda=g_{s} \mu_{B} G_{m} a_{0} / \hbar$, where $a_{0}=\sqrt{\hbar / 2 m \omega_{m}}, g_{s} \simeq 2$ is the Landé g-factor, $\mu_{B}$ is the Bohr magneton, and $G_{m}$ is the magnetic field gradient along the NV center axis.

In order to prepare the Fock states, we first cool the mechanical mode to the ground state with sideband cooling [16], or spin assistant phonon cooling [59]. The NV spin is initially set to the state $|0\rangle$, which is decoupled from the mechanical mode during the cooling. Initialization and single shot detection of the NV spin have been well accomplished experimentally [61]. We assume that the NV center is at a position with zero magnetic field and a large field gradient. We apply a microwave drive with the Hamiltonian $H_{\text {drive }}=\hbar\left(\Omega_{\mathrm{NV},+1} e^{i \omega_{l+} t}|0\rangle\langle+1|+\right.$ $\Omega_{\mathrm{NV},-1} e^{i \omega_{l-t}}|0\rangle\langle-1|+$ h.c. $) / 2$ and set the Rabi frequency $\Omega_{\mathrm{NV}, \pm 1}=\Omega_{\mathrm{NV}}$ and the detuning $\Delta_{ \pm} \equiv \omega_{l \pm}-\omega_{ \pm 1}=\Delta$. With $\Delta \gg\left|\Omega_{\mathrm{NV}}\right|$, we adiabatically eliminate the level $|0\rangle$ and get the following effective Hamiltonian

$$
H_{e}=\hbar \omega_{m} a_{m}^{\dagger} a_{m}+\hbar \Omega \sigma_{z}+\hbar \lambda\left(\sigma_{+}+\sigma_{-}\right)\left(a_{m}+a_{m}^{\dagger}\right)
$$

where $\Omega=\left|\Omega_{\mathrm{NV}}\right|^{2} / 4 \Delta, \sigma_{z}=|+\rangle\langle+|-|-\rangle\langle-|, \sigma_{+}=$ $|+\rangle\left\langle-\left|, \sigma_{-}=\right|-\right\rangle\langle+|$, and we have defined the new basis states $|+\rangle=(|+1\rangle+|-1\rangle) / \sqrt{2},|-\rangle=(|-1\rangle-|-1\rangle) / \sqrt{2}$. In the limit $\lambda \ll \omega_{m}$, we set $\Omega=\omega_{m} / 2$ and use the rotating wave approximation to get an effective interaction Hamiltonian between the mechanical mode and the NV center spin, with the form $H_{J C}=\hbar \lambda \sigma_{+} a_{m}+$ h.c.. This represents the standard Jaynes-Cummings(J-C) coupling Hamiltonian. Similarly, if we set $\Omega=-\omega_{m} / 2$, the anti J-C Hamiltonian can be realized with $H_{a J C}=\hbar \lambda \sigma_{+} a_{m}^{\dagger}+$ h.c.. 
Arbitrary Fock states and their superpositions can be prepared with a combination of $\mathrm{J}-\mathrm{C}$ and anti J-C coupling Hamiltonians. For example, to generate the Fock state $|2\rangle_{m}$, we initialize the state to $|+\rangle|0\rangle_{m}$, turn on the $\mathrm{J}$-C coupling for a duration $t_{1}=\pi /(2 \lambda)$ to get $|-\rangle|1\rangle_{m}$, and then turn on the anti J-C coupling for a duration $t_{2}=t_{1} / \sqrt{2}$ to get $|+\rangle|2\rangle_{m}$. The Fock state with arbitrary phonon number $n_{m}$ can be generated by repeating the above two basic steps, and the interaction time is $t_{i}=t_{1} / \sqrt{i}$ for the $i$ th step [62]. Superpositions of different Fock states can also be generated. For instance, if we initialize the state to $\left(c_{0}|+\rangle+c_{1}|-\rangle\right) \otimes|0\rangle_{m} / \sqrt{2}$ through a microwave with arbitrary coefficients $c_{0}, c_{1}$, and turn on the J-C coupling for a duration $t_{1}$, we get the superposition state $|-\rangle \otimes\left(c_{1}|0\rangle_{m}+i c_{0}|1\rangle_{m}\right) / \sqrt{2}$.Using the optical cavity, the Fock state $\left|n_{m}\right\rangle_{m}$ of mechanical mode can also be mapped to the corresponding Fock state of the output light field [27].

The effective Hamiltonian for the spin-phonon coupling takes the form $H_{Q N D}=\hbar \chi \sigma_{z} a_{m}^{\dagger} a_{m}$ with $\chi=$ $4 \Omega \lambda^{2} /\left(4 \Omega^{2}-\omega_{m}^{2}\right)$ when the detuning ||$\Omega\left|-\omega_{m} / 2\right| \gg \lambda$. The Hamiltonian $H_{Q N D}$ can be used for a quantum non-demolition measurement(QND) measurement of the phonon number: we prepare the NV center spin in a superposition state $\left.|+\rangle+e^{i \phi}|-\rangle\right) / \sqrt{2}$, and the phase $\phi$ evolves by $\phi(t)=\phi_{0}+2 \chi n_{m} t$, where $n_{m}=a_{m}^{\dagger} a_{m}$ denotes the phonon number. Through a measurement of the phase change, one can detect the phonon number.

The preparation and detection of the Fock states can all be done within the spin coherence time. Let us estimate the typical parameters. A large magnetic field gradient can be generated by moving the nanodiamond close to a magnetic tip. Here we take the gradient $G=10^{5} \mathrm{~T} / \mathrm{m}$ and get the coupling $\lambda \simeq 2 \pi \times 52 \mathrm{kHz}$ for a nanodiamond with the diameter $d=30 \mathrm{~nm}$ in an optical trap with a trapping frequency $\omega_{m}=2 \pi \times 0.5 \mathrm{MHz}$. The Fock states and their superpositions can then be generated with a time scale $1 / \lambda$ about a few $\mu$ s, and the QND detection rate $2|\chi| \sim 2 \pi \times 25 \mathrm{kHz}$ with the detuning ||$\Omega\left|-\omega_{m} / 2\right| \sim 5 \lambda$. The NV electron spin dephasing time over $1.8 \mathrm{~ms}$ has been observed at room temperature [63], which is long compared with the Fock state preparation time $1 / \lambda$ and the detection time $1 /(2|\chi|)$.

\section{Schödinger's cat states}

Creating Shrödinger's cat states with massive objects is one of the most challenging and attractive goals in macroscopic quantum mechanics. [6 8]. To generate spatial quantum superpositions and other non-Gaussian states with an optical cavity, however, requires a very strong quadratic coupling [28, 29, 64]. This is a very demanding requirement. To enhance the quadratic coupling, Romero-Isart et al. 28] proposed to prepare spatial quantum superpositions of nanoparticles with two inter-connected high-finesse optical cavities: one cavity for ground state cooling, and the other cavity for prepar- (a)

$\downarrow$

(b)

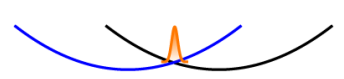

(c)

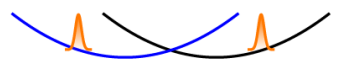

FIG. 9: (a) Maximum spatial separation $D_{m}$ of the superposition state as a function of trap frequency $\omega_{m 2}$ when the magnetic gradient is $10^{5} \mathrm{~T} / \mathrm{m}$. (b) Maximum spatial separation $D_{m}$ as a function of the magnetic gradient $G$ when the trapping frequency is $1 \mathrm{kHz}$. Macroscopic superposition states with separation larger than the size of the particle can be achieved with a moderate magnetic gradient.

ing the superposition state with a squared position measurement when the nanoparticle falls through it. The Schödinger's cat state can also be generated by ultraviolet (UV) laser if the ground state is reached [34]. Then, we turn off the optical trap, and let the wavefunction expand for a time $t_{1}$. A tightly focused UV laser pulse is shot through the center of the expanded wavefunction, whose scale is in the order of hundreds nanometers. The Schrödinger's cat state will generate conditional on no light being scattered. We can repeat the procedures until Schrödinger's cat state is generated.

Here we discuss how to create the Schödinger's cat state with a levitated nanodiamond with a NV center [54]. As we discussed in the previous subsection, the strong nonlinear coupling can be realized in the nanocrystal diamond with building in NV centers. Therefore, in this system, spatial quantum superpostion state, or Schrödinger's cat state, can be easily generated without measurement. Without the microwave driving, the spinmechanical coupling Hamiltonian takes the form

$$
H=\hbar \omega_{m} a_{m}^{\dagger} a_{m}+\hbar \lambda S_{z}\left(a_{m}+a_{m}^{\dagger}\right) .
$$

The mechanical mode is initialized to the vacuum state $|0\rangle_{m}$ (or a Fock state $\left|n_{m}\right\rangle_{m}$ ) in a strong trap with the trapping frequency $\omega_{m 0}$ and the NV center spin is prepared in the state $|0\rangle$. Although the ground state cooling is most effective in a strong trap, to generate large spatial separation of the wave packets it is better to first lower the trap frequency by tuning the laser intensity for the optical trap. While it is possible to lower the trap frequency through an adiabatic sweep to keep the phonon state unchanged, a more effective way is to use a non-adiabatic state-preserving sweep [65], which allows arbitrarily short sweeping time. We denote $\left|n_{m}\right\rangle_{m 1}$ as the mechanical state in the lower frequency $\omega_{m 1}$.

We then apply an impulsive microwave pulse to suddenly change the NV spin to the state $(|+1\rangle+|-1\rangle) / \sqrt{2}$ and simultaneously decrease the trap frequency to $\omega_{m 2} \leq$ $\omega_{m 1}$. The evolution of the system state under the Hamil- 
tonian (Eq. 13) then automatically split the wave packet for the CoM motion of the nanodiamond (see the illustration in Fig. 9). The splitting attains the maximum at time $T_{2} / 2=\pi / \omega_{m 2}$, where the maximum distance of the two wave packets in the superposition state is $D_{m}=8 \lambda a_{2} / \omega_{m 2}=4 g_{s} \mu_{B} G /\left(m \omega_{m 2}^{2}\right)$, where $a_{2}=$ $\sqrt{\hbar / 2 m \omega_{m 2}}$. At this moment, the system state is $\left|\Psi_{S}\right\rangle=$ $\left(|+1\rangle\left|D_{m} / 2\right\rangle_{n_{m}}+|-1\rangle\left|-D_{m} / 2\right\rangle_{n_{m}}\right) / \sqrt{2}$, where $\mid \pm$ $\left.D_{m} / 2\right\rangle_{n_{m}} \equiv(-1)^{a_{m}^{\dagger} a_{m}} \exp \left[ \pm D_{m}\left(a_{m}^{\dagger}-a_{m}\right) / 4 a_{2}\right]\left|n_{m}\right\rangle_{1}$ is the displaced Fock state (or coherent states when $n_{m}=$ 0 ). This is just the entangled spatial superposition state. To transform the entangled cat state $\left|\Psi_{S}\right\rangle$ to the standard cat state $\left|\psi_{ \pm}\right\rangle_{n_{m}} \equiv\left(\left|D_{m} / 2\right\rangle_{n_{m}} \pm\left|-D_{m} / 2\right\rangle_{n_{m}}\right) / \sqrt{2}$, we need to apply a disentangling operation to conditionally flip the NV spin using displacement of the diamond as the control qubit. This can be achieved as different displacements of the wavepacket induce relative energy shifts of the spin levels due to the applied magnetic field gradient. As an estimate, for the example that we consider a $30 \mathrm{~nm}-$ diameter diamond in a $20 \mathrm{kHz}$ trap under a magnetic gradient of $3 \times 10^{4} \mathrm{~T} / \mathrm{m}$, the spin energy splitting is about 2.4 $\mathrm{MHz}$ between the $|+1\rangle\left|D_{m} / 2\right\rangle_{n_{m}}$ and $|-1\rangle\left|-D_{m} / 2\right\rangle_{n_{m}}$ components, which is much larger than the typical transition linewidth of the NV spin (in the order of $\mathrm{kHz}$ ). So we can apply first an impulsive microwave pulse to transfer the component state $|+1\rangle\left|D_{m} / 2\right\rangle_{n_{m}}$ to $|0\rangle\left|D_{m} / 2\right\rangle_{n_{m}}$ without affecting $|-1\rangle\left|-D_{m} / 2\right\rangle_{n_{m}}$ and then another pulse to transfer $|-1\rangle\left|-D_{m} / 2\right\rangle_{n_{m}}$ to $\pm|0\rangle\left|-D_{m} / 2\right\rangle_{n_{m}}$. After the two pulses, the spin state gets disentangled and the position of the diamond is prepared in the quantum superposition state $\left|\psi_{ \pm}\right\rangle_{n_{m}}$.

To detect spatial superposition state, we can turn off the optical trap and let the spatial wave function freely evolve for some time $t$. The split wave packets will interference just like the Young's double slit experiment. The period of the interference pattern is $\Delta z=2 \pi \hbar t /\left(m D_{m}\right)$. As an estimation of typical parameters, we take $\omega_{m 1}=$ $\omega_{m 2}=2 \pi \times 20 \mathrm{kHz}, d=30 \mathrm{~nm}$, and magnetic field gradient $3 \times 10^{4} \mathrm{~T} / \mathrm{m}$. The spin-phonon coupling rate $\lambda \simeq 2 \pi \times 77 \mathrm{kHz}$ and the maximum distance $D_{m} \simeq 31 a_{2}$. The preparing time of sperposition state is about $25 \mu \mathrm{s}$, which is much less than the coherence time of the NV spin. For the time of flight measurement after turn-off of the trap, we see the interference pattern with a period of $47 \mathrm{~nm}$ after $t=10 \mathrm{~ms}$, which is large enough to be spatially resolved [18, 19, 22].

\section{ULTRASENSITIVE FORCE DETECTION}

In an ultra-high vacuum environment, the CoM motion of optically levitated sensors experiences minimal dissipation, enabling ultra-sensitive force detection [27, 31, 66, 67]. Unlike conventional sensors consisting of solid-state mechanical resonators, e.g. cantilevers or membranes, the CoM motion of optically trapped dielectric objects is immune to the chief sources of dissipation in these devices at low pressure, consisting of lossy internal flexural and vibrational modes, surface imperfections, and clamping mechanisms. The result is sub-attonewton force sensitivity that may have a number of applications ranging from Casimir force measurements, experimental gravitation, electric or magnetic field sensing, single molecules detecting, to inertial sensing.

\section{A. Force sensing with mechanical oscillators.}

High force sensitivity resonant sensors have typically consisted of solid-state micro-fabricated structures, for example cantilever beams or membranes 66, 68]. The achievement of aN/ $\mathrm{Hz}^{1 / 2}$ sensitivity in cryogenic cantilevers has lead to magnetic resonance force microscopy with the sensitivity to detect single electron spins in solids [69], and has allowed sensitive tests for nonNewtonian gravity at the $\sim 10 \mu \mathrm{m}$ length scale [70]. In these systems, the internal materials losses and clamping mechanisms are responsible for limiting the quality factor of the oscillator to typically below $Q \sim 10^{6}$. For force detection, it is desirable to have minimal dissipation, as the minimum detectable force due to thermal noise scales as $Q^{-1 / 2}$. For a harmonic oscillator with natural frequency $\omega_{0}$ it can be expressed as

$$
F_{\min }=\left[4 k k_{B} T b / \omega_{0} Q\right]^{1 / 2}
$$

where $b$ is the bandwidth of the measurement, $T$ is the effective temperature of the mode under consideration, and $k$ is the spring constant. In ultra-high vacuum, the CoM motion of optically levitated micron-sized dielectric spheres and could exhibit $Q$ factors approaching $10^{12}$, leading to force sensitivity well below $1 \mathrm{aN} / \mathrm{Hz}^{1 / 2}$ at room temperature. Experiments performed thus far have achieved inferred force sensitivities at the level of $\sim 10^{-20} \mathrm{~N} / \mathrm{Hz}^{1 / 2}$ for a $70 \mathrm{~nm}$ particle at $P=10^{-5} \mathrm{mbar}$ in Ref. [22], and of order $10^{-19} \mathrm{~N} / \mathrm{Hz}^{1 / 2}$ for a $3 \mu \mathrm{m}$ diameter sphere feedback-cooled to $1.5 \mathrm{mK}$ at $5.2 \mathrm{mPa}$ in Ref. [19].

For a particle of mass $m$ in an optical trap, we can rewrite Eq. (14) as $F_{\min }=\left[4 k_{B} T m b \gamma_{g}\right]^{1 / 2}$, where the background gas collision has a loss rate of $\gamma_{g}=$ $16 P_{\text {gas }} /(\pi \bar{v} \rho a)$ [71], for a background air pressure of $P_{\text {gas }}$ and rms gas velocity $\bar{v}$, and a sphere of radius $a$ and density $\rho$. However, as we discuss below, this formula must be modified due to heating by the recoil of scattered trap laser photons. Such scattering produces a heating rate $\gamma_{s c}=\frac{2}{5} \frac{\pi^{2} \omega_{0} V}{\lambda^{3}} \frac{(\epsilon-1)}{(\epsilon+2)}$, where $V$ is the sphere volume, $\lambda$ is the trap laser wavelength, and $\epsilon$ is the real part of the dielectric function for the sphere.

Laser cooling is essential for operation in high vacuum for several reasons. A mechanical oscillator with frequency $\sim 100 \mathrm{kHz}$ and $Q=10^{12}$ will respond to perturbations with a characteristic time scale of $2 Q / \omega_{0} \approx$ $3 \times 10^{6} \mathrm{~s}$, which is not practical for laboratory measurement. The cooling thus serves to damp the motion of 
the oscillator so that perturbations to the system can ring-down within reasonably short periods of time. In addition, as the laser intensity determines the trapping frequency, it must be stabilized if the particle is to remain on resonance in the case of resonant detection. By also damping the oscillator, the laser cooling can therefore significantly reduce the requirement on the laser intensity stabilization. Finally, the cooling is necessary to mitigate heating due to the recoil of trap laser photons. Such recoil heating leads to a momentum diffusion process, which left unchecked, can result in heating of the CoM motion of the particle and its eventual loss. This heating modifies the expected form of the thermal noise limited force sensitivity.

Either (active) feedback cooling or (passive) cavity cooling serves to damp the $Q$ factor to $Q_{\text {eff }}$ while at the same time the mode temperature is reduced to $T_{\text {eff }}$. The force sensitivity scales as $\sqrt{T_{\text {eff }} / Q_{\text {eff }}}$ : the minimum detectable force due to thermal noise at temperature $T_{\text {eff }}$ is $F_{\text {min }}=\sqrt{\frac{4 k k_{B} T_{\text {eff }} b}{\omega_{0} Q_{\text {eff }}}}$, where $k$ is the CoM mode spring constant, and $b$ is the bandwidth of the measurement.

For example, in the case of cavity-cooling, the thermalnoise limited minimum detectable force becomes

$$
F_{\min }=\sqrt{4 k_{B} T m b \gamma_{g}\left[1+\frac{\gamma_{\mathrm{sc}}+R_{+}}{n_{i} \gamma_{g}}\right]} .
$$

We can define a factor $\chi=\frac{\gamma_{\mathrm{sc}}+R_{+}}{n_{i} \gamma_{g}}$ which describes the importance of photon recoil heating $\gamma_{\mathrm{sc}}$ and the efficiency of the cavity cooling. Here $n_{i} \equiv k_{B} T / \hbar \omega_{0}$ is the initial mean phonon occupation number. The factor $R_{+}$, defined in Ref. [16] can be minimized by going into the resolved sideband regime and can be generally neglected when compared with $\gamma_{s c}$. There are two general regimes of scaling, $\chi<<1$ and $\chi>>1$. For $\chi<<1$, the effects of photon recoil do not significantly degrade the force sensitivity, and $F_{\min } \propto a T^{1 / 4} P^{1 / 2}$ and is independent of trap frequency. In the regime $\chi>>1$, photon recoil heating becomes significant, and damping without an equal amount of cooling occurs. Here for a nanosphere the sensitivity scales as $F_{\min } \propto \omega_{0} a^{3}$ and is independent of $T$ and $P$. A micro-disc geometry scatters much less light, as pointed out in Ref. [72] and recoil heating is significantly reduced. Also the micro-disc allows a larger mass to be trapped and localized in a particular anti-node of the standing wave in the cavity which is advantageous e.g. for gravitational wave strain sensitivity 33.

In Fig. 10 we show the dependence of the force and acceleration sensitivity on the radius of the sphere at fixed trapping frequency of $1 \mathrm{kHz}$ assuming $R_{+}<<\gamma_{s c}$, at $T=300 \mathrm{~K}$ and $P=10^{-10}$ torr. At larger radii the regime $\chi>>1$ is realized, with the minimum detectable force scaling as the sphere volume. In Fig. 11 we show the dependence of the force sensitivity on trapping frequency for the fixed size $a=150 \mathrm{~nm}$. As the trapping frequency increases, the linear scaling with $\omega_{0}$ is apparent as the regime $\chi>>1$ is realized.

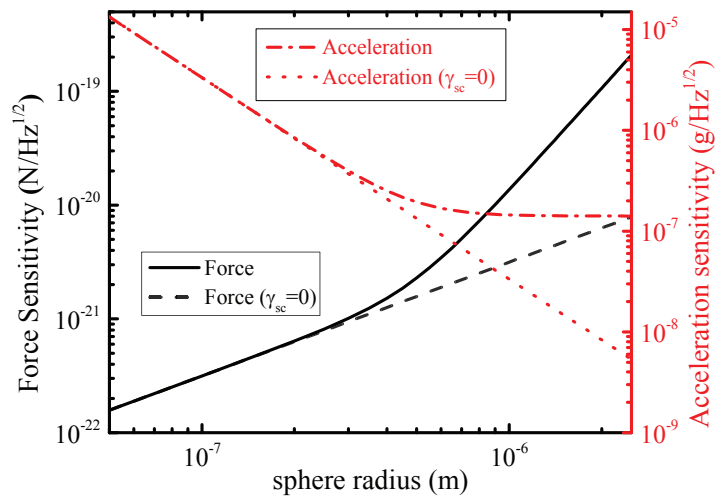

FIG. 10: Thermal noise limited force sensitivity and acceleration sensitivity for an optically trapped silica microsphere at pressure $P=10^{-10}$ Torr and $T=300 \mathrm{~K}$ versus sphere radius at $1 \mathrm{kHz}$ trap frequency. The deviation from the scaling in Eq. (14) is due to photon recoil heating.

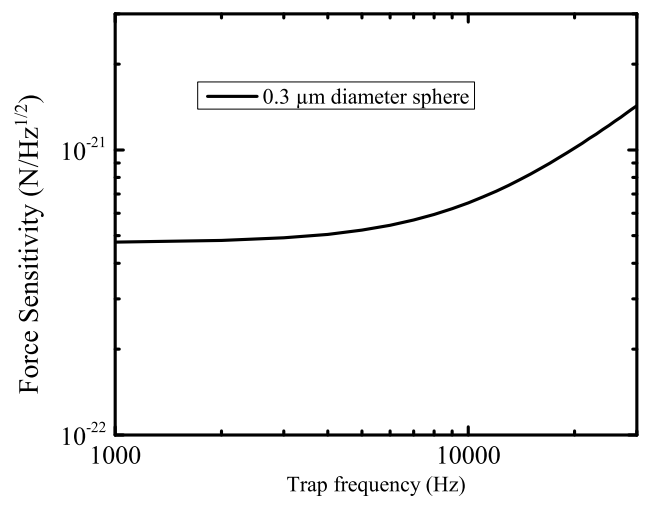

FIG. 11: Scattering-limited sensitivity for a $0.3 \mu \mathrm{m}$ diameter bead vs. trapping frequency for planned experimental parameters.

\section{B. Applications.}

For the following discussion, we consider a dielectric sphere optically trapped and cooled in a cavity using two light fields of wavevector $k_{t}=2 \pi / \lambda_{\text {trap }}$ and $k_{c}=2 \pi / \lambda_{\text {cool }}$, respectively. The sphere is levitated in an anti-node of the trapping light which can be located near one of the mirrors of the cavity at frequencies ranging from $\sim 1-100 \mathrm{kHz}$. The Gaussian profile of the trapping beam near the mode waist provides transverse confinement. Cooling of the transverse motion can be done with active feedback to modulate the power of a transverse cooling laser using the signal from a transverse position measurement.

For detecting the position of the sensor, the phase of 
the cooling light reflected from the cavity is modulated through the optomechanical coupling $\partial \omega_{c} / \partial z=k_{c} g$. Photon shot-noise limits the minimum detectable phase shift to $\delta \phi \approx 1 /(2 \sqrt{I})$ where $I \equiv P_{c} /\left(\hbar \omega_{c}\right)[73$. The corresponding photon shot-noise limited displacement sensitivity is $\sqrt{S_{z}(\omega)}=\frac{\kappa}{4 k_{c} g} \frac{1}{\sqrt{I}} \sqrt{1+\frac{4 \omega^{2}}{\kappa^{2}}}$ [74], for an impedance matched cavity. Here $P_{c}$ and $\omega_{c}$ are the cooling laser power and frequency, $g=\frac{3 V}{4 V_{c}} \frac{\epsilon-1}{\epsilon+2} \omega_{c}$, and $\kappa$ is the optical cavity loss rate. The cavity mode volume is $V_{c}$. The thermally-driven resonant motion of the sensor is typically much greater than the photon shot noise limited detection floor due to the high $Q_{\text {eff }}$.

\section{Short-distance tests of gravity.}

By trapping a nanosphere at an anti-node located at sub-micron distance from one of the cavity mirrors, it is possible to realize an experiment for testing gravity at the micron length scale [31]. Non-Newtonian gravity-like forces can be tested by monitoring the displacement of the sphere as a mass is brought behind the cavity mirror. Short-range corrections to Newtonian gravity are generally parameterized according to a Yukawa-type potential

$$
V=-\frac{G_{N} m_{1} m_{2}}{r}\left[1+\alpha e^{-r / \lambda}\right]
$$

where $m_{1}$ and $m_{2}$ are two masses interacting at distance $r, \alpha$ is the strength of the correction relative to gravity, and $\lambda$ is the range of the interaction. For two masses with density $\rho$ and linear dimesion $\lambda$ that are separated by $r \approx \lambda$, a Yukawa-force scales roughly as $F_{Y} \sim G_{N} \rho^{2} \alpha \lambda^{4}$, rapidly decreasing with smaller $\lambda$. For a gold masss, for an interaction potential with $\alpha=10^{5}$ and $\lambda=1 \mu \mathrm{m}, F_{Y} \sim 10^{-21}$ N. As the thermal-noiselimited force sensitivity of micron-size trapped spheres can be of order $\sim 10^{-21} \mathrm{~N} / \sqrt{\mathrm{Hz}}$, this setup therefore allows probing deep into unexplored regimes. For instance, current experimental limits at $\lambda=1 \mu \mathrm{m}$ have only ruled out interactions with $|\alpha|$ exceeding $10^{10}$ [31, 75 77].

\section{Casimir Forces.}

The Casimir Effect [78] is a macroscopic manifestation of quantum vacuum fluctuations, and is a testament to the theory of quantum electrodynamics, arguably the most accurately known theory in physics. At the same time, developing our understanding of it is becoming essential for pushing the size limits in nanotechnology and nano-electro-mechanical systems. One of the most widely studied geometries involves the Casimir interaction between a sphere and plane. Previous measurements have been performed in the limit that their separation distance $d$ is small compared with the sphere radius a 79 83. On the other hand, the Casimir-polder limit has also been explored using cold atoms, where the atomic size is much smaller than their distance to the plane [84]. However, there is a completely unexplored intermediate regime where the size of the sphere is on the order of the sphere-surface separation. Such a regime poses an experimental challenge for commonly used measurement approaches involving a sphere attached to a torsional resonator the mechanical resonator which is used for force sensing is tethered to the sphere, and therefore affects the geometry once the separation distance approaches the size of the sphere. By using an optically-trapped nanosphere as the force sensor, one inherently overcomes this difficulty. With a sphere trapped in an anti-node close to an end-mirror of the cavity, Casimir forces due to the metallic end-mirror can be measured as a frequency shift of the oscillator. This type of experiment could allow a pristine dielectric-sphere/metal-plate geometry to be explored over a range of distances, from the short range limit where the proximity force approximation (PFA) is valid and the force varies as $1 / d^{3}$, to the long range $1 / d^{5}$ Casimir-polder limit [31].

\section{Gravitational Waves.}

Nano- and micro-scale dielectric sensors trapped inside a medium-finesse optical cavity can be used to detect high frequency gravitational wave (GW) radiation 33. The direct detection of gravitational radiation is very likely to occur in the next decade with the new generation of laser-interferometer gravitational wave observatories [85 89]. While these detectors have been optimized in the frequency band of $10-10^{4} \mathrm{~Hz}$, their sensitivity decreases at higher frequency due to photon shot noise. The optically trapped sensor offers improved sensitivity in the frequency range of $50-300 \mathrm{kHz}$ using an approach that does not rely on a shot-noise limited displacement measurement of test mass mirrors, but rather depends on a precision force measurement on the resonant harmonically trapped sensor. The detector can yield sensitivities improved by more than an order of magnitude in this frequency band when compared with existing interferometers, while being only a fraction of their size. The approach extends the effective search volume for sources between 100 and $300 \mathrm{kHz}$ by $\sim 10-10^{3}$ when compared with Advanced LIGO [86]. At such high frequencies, there may be sources of gravitational radiation from physics beyond the standard model. One example may result from the effects of the QCD axion on stellar mass black holes $(\mathrm{BHs})$ through $\mathrm{BH}$ superradiance [90]. This novel signal comes from axion annihilation to gravitons and is monochromatic and long-lived.

In the approach proposed in Ref. [33], a dielectic nanosphere or microdisc is optically trapped in an antinode of a cavity of length at a position close to the input mirror. A second light field with two different frequency components is used to cool and read out the axial position of the levitated object, respectively. A passing gravitational wave displaces the sensor from its equilibrium 
position in the cavity, resulting in a measurable displacement of the levitated object. Gravitational wave strain sensitivity can approach $\sim 10^{-22} / \sqrt{\mathrm{Hz}}$ for frequencies near $100 \mathrm{kHz}$ for micron-sized discs in a cavity of length $100 \mathrm{~m}$. The resulting displacement of the sensor is resonantly enhanced when the frequency of the gravitational wave coincides with the optical trap frequency.

\section{Detecting single molecule collisions}

Detection of individual collisions between single molecules and the nanosphere would lead to a test of the Maxwell-Boltzmann distribution on single-collision level. By using the 3D cooling sideband cooling scheme, we may archive it by detecting the output light pulses. Considering the gas pressure $P$ at temperature $T_{\text {env }}$, the radius of the sphere $r$, the molecule mass $m_{m}$, we have the collision number per second $N=\left(2 \pi r^{2}\right) P / \sqrt{\pi m_{m} k_{B} T_{\text {env }} / 2}$, where $k_{B}$ is the Boltzmann constant. The collision time is estimated to be much less than the nanosphere oscillation time scale. The three phonon modes initially in vacuum will be in a state with mean phonon number $n_{j 0}:\left\langle a_{j}^{\dagger}\left(t_{0}\right) a_{j}\left(t_{0}\right)\right\rangle=n_{j 0}$ after a single collision, where $t_{0}$ is the time when collision happens. For this case, the output field is

$$
a_{c j}^{\text {out }}(t)=-i \frac{2 g \alpha_{j}}{\sqrt{\kappa_{j}}} \exp \left[-\frac{2 g_{j}^{2}\left|\alpha_{j}\right|^{2}}{\kappa_{j}}\left(t-t_{0}\right)\right] a_{j}\left(t_{0}\right)+a_{c j}^{\text {in }},
$$

It is easy to find that $\int_{t_{0}}^{\infty}\left\langle a_{c j}^{\text {out }}(t) a_{c j}^{\text {out } \dagger}(t)\right\rangle d t=n_{j 0}$. This implies that the output-pulse photon number is equal to the increase of the phonon number after the collision. From above discussion, we get the phonon decay time $\tau_{j}=\kappa_{j} /\left(4 g_{j}^{2}\left|\alpha_{j}\right|^{2}\right)$, which is also the pulse duration of the output light of mode $a_{c j}$. The phonon number can be measured by detecting the output light pulse. Therefore, $\tau_{j}$ is the measurement time for the phonon mode $a_{j}$ after the collision. Therefore, as long as $\tau_{j} \ll 1 / N$, the collision events can be measured individually.
Moreover, to make detecting efficiency high, the phonon number after the collision requires to be more than one. For the first case, we suppose the collision is completely elastic. The average increase of the phonon number for $a_{j}$ is $n_{j 0}=2 m_{m}^{2}\left\langle v_{j}^{2}\right\rangle /\left(\hbar \omega_{j} m\right)$ with $\left\langle v_{j}^{2}\right\rangle$ the the mean velocity square along the axis $q_{j}$. As a result, the requirement for the phonon number change could be rewritten as $2 k_{B} T_{\text {env }}>\hbar \omega_{j}\left(m / m_{m}\right)$. If the collision is completely inelastic, the molecule will attach on the surface of the nanosphere for a while before being kicked out. The output velocity distribution is completely determined by the temperature of the nanosphere surface. The criteria should be either $k_{B} T_{\text {env }}>2 \hbar \omega_{j}\left(m / m_{m}\right)$, or $k_{B} T_{\text {sur }}>2 \hbar \omega_{j}\left(m / m_{m}\right)$, where $T_{\text {sur }}$ is the temperature of the surface of the nanosphere. To distinguish elastic and inelastic collision, we can cool the temperature to the limit that $k_{B} T_{\text {env }} \ll \hbar \omega_{j}\left(m / m_{m}\right)$, and makes the condition $k_{B} T_{\text {sur }}>2 \hbar \omega_{j}\left(\mathrm{~m} / \mathrm{m}_{\mathrm{m}}\right)$ fulfills by adding a long wavelength laser to heat the sphere. If the collisions are all elastic, there is no signal on the photon detectors. If there are parts of the collisions are inelastic, there are output pulses of lights. Besides, the distribution of the photon numbers is determined by the surface temperature of the sphere. In other words, we can measure the surface temperature of the nanosphere by detecting the output light pulses.

\section{Other applications.}

By carrying a non-zero net electric charge, an optically trapped dielectric sphere becomes a sensitive detector for electric fields. For a charged sphere of diameter $300 \mathrm{~nm}$ with an electric field of $\sim 10^{7} \mathrm{~V} / \mathrm{m}$ at its surface, a $10^{-21}$ $\mathrm{N} / \sqrt{\mathrm{Hz}}$ sensitivity corresponds to an electric field sensitivity of $\sim 10 \mu \mathrm{V} / \mathrm{m} / \sqrt{\mathrm{Hz}}$. Correspondingly if the sphere were functionalized with a magnetic moment, sensitive magnetic field sensing may be possible, for example enabling magnetic resonance force microscopy [69].
[1] R Penrose. On gravity's role in quantum state reduction. Gen. Rel. Grav., 28(5), 1572 (1996).

[2] J. Christian. Testing gravity-driven collapse of the wave function via cosmogenic neutrinos. Phys. Rev. Lett. 95, 160403 (2005).

[3] J. van Wezel, T. Oosterkamp, and J. Zaanen. Towards an experimental test of gravity-induced quantum state reduction. Phil. Mag. 88, 1005 (2008).

[4] L. Diósi. Models for universal reduction of macroscopic quantum fluctuations. Phys. Rev. A 40, 1165 (1989).

[5] G. C. Ghirardi, A. Rimini, and T. Weber. Unified dynamics for microscopic and macroscopic systems. Phys. Rev. D 34, 470 (1986).

[6] A. Bassi, K. Lochan, S. Satin, T. P. Singh, and H. Ulbricht. Models of wave-function collapse, underlying the- ories, and experimental tests. Reviews of Modern Physics 85, 471-527 (2013).

[7] Stefan Nimmrichter and Klaus Hornberger. Macroscopicity of mechanical quantum superposition states. Phys. Rev. Lett. 110, 160403 (2013).

[8] Y. Chen. Macroscopic quantum mechanics: theory and experimental concepts of optomechanics. Journal of Physics B Atomic Molecular Physics 46, 104001 (2013).

[9] M. Aspelmeyer, S. Groeblacher, K. Hammerer, and N. Kiesel. Quantum optomechanics - throwing a glance. J. Opt. Soc. Am. B 27, A189-A197 (2010).

[10] I. Wilson-Rae, N. Nooshi, W. Zwerger, and T. J. Kippenberg. Theory of Ground State Cooling of a Mechanical Oscillator Using Dynamical Backaction. Phys. Rev. Lett. 99(9), 093901 (2007). 
[11] F. Marquardt, J. P. Chen, A. A. Clerk, and S. M. Girvin. Quantum Theory of Cavity-Assisted Sideband Cooling of Mechanical Motion. Phys. Rev. Lett. 99(9), 093902 (2007).

[12] J. Chan, T. P. M. Alegre, A. H. Safavi-Naeini, J. T. Hill, A. Krause, S. Gröblacher, M. Aspelmeyer, and O. Painter. Laser cooling of a nanomechanical oscillator into its quantum ground state. Nature 478, 89-92 (2011).

[13] J. D. Teufel, T. Donner, D. Li, J. W. Harlow, M. S. Allman, K. Cicak, A. J. Sirois, J. D. Whittaker, K. W. Lehnert, and R. W. Simmonds. Sideband cooling of micromechanical motion to the quantum ground state. $\mathrm{Na}$ ture, 475, 359-363 (2011).

[14] AD O'Connell and et al. Quantum ground state and single-phonon control of a mechanical resonator. Nature 464, 697-703 (2010).

[15] M. Aspelmeyer, T. J. Kippenberg, and F. Marquardt. Cavity Optomechanics. ArXiv e-prints 1303.4976, March 2013.

[16] D. E. Chang and et al. Cavity opto-mechanics using an optically levitated nanosphere. PNAS 107(3), 1005-1010 (2010).

[17] O. Romero-Isart, ML Juan, R. Quidant, and JI Cirac. Towards quantum superposition of living organisms. New J. Phys. 12, 033015 (2010).

[18] Tongcang Li, Simon Kheifets, David Medellin, and Mark G. Raizen. Measurement of the Instantaneous Velocity of a Brownian Particle. Science, 328, 1673 (2010).

[19] T. Li, S. Kheifets, and M. G. Raizen. Millikelvin cooling of an optically trapped microsphere in vacuum. Nature Physics 7, 527-530 (2011).

[20] P. F. Barker and M. N. Shneider. Cavity cooling of an optically trapped nanoparticle. Phys. Rev. A 81(2), 023826 (2010).

[21] P. F. Barker. Doppler Cooling a Microsphere. Phys. Rev. Lett. 105(7), 073002 (2010).

[22] J. Gieseler, B. Deutsch, R. Quidant, and L. Novotny. Subkelvin Parametric Feedback Cooling of a LaserTrapped Nanoparticle. Phys. Rev. Lett. 109(10), 103603 (2012).

[23] N. Kiesel, F. Blaser, U. Delic, D. Grass, R. Kaltenbaek, and M. Aspelmeyer. Cavity cooling of an optically levitated nanoparticle. arXiv e-prints 1304.6679, 2013.

[24] P. Asenbaum, S. Kuhn, S. Nimmrichter, U. Sezer, M. Arndt. Cavity cooling of free silicon nanoparticles in high-vacuum. arXiv e-prints 1306.4617, 2013.

[25] T S Monteiro et al. Dynamics of levitated nanospheres: towards the strong coupling regime. New J. Phys. 15, 015001 (2013).

[26] Zhang-qi Yin. Phase noise and laser-cooling limits of optomechanical oscillators. Phys. Rev. A 80(3), 033821 (2009).

[27] Z.-Q. Yin, T. Li, and M. Feng. Three-dimensional cooling and detection of a nanosphere with a single cavity. Phys. Rev. A 83(1), 013816 (2011).

[28] O. Romero-Isart, A. C. Pflanzer, M. L. Juan, R. Quidant, N. Kiesel, M. Aspelmeyer, and J. I. Cirac. Optically levitating dielectrics in the quantum regime: Theory and protocols. Phys. Rev. A 83(1), 013803 (2011).

[29] O. Romero-Isart, A. C. Pflanzer, F. Blaser, R. Kaltenbaek, N. Kiesel, M. Aspelmeyer, and J. I. Cirac. Large Quantum Superpositions and Interference of Massive Nanometer-Sized Objects. Phys. Rev. Lett. $\mathbf{1 0 7}(2), 020405$ (2011).

[30] O. Romero-Isart. Quantum superposition of massive objects and collapse models. Phys. Rev. A 84(5), 052121 (2011).

[31] Andrew A. Geraci, Scott B. Papp, and John Kitching. Short-range force detection using optically cooled levitated microspheres. Phys. Rev. Lett. 105(10), 101101 (2010).

[32] Wenjie Nie, Yueheng Lan, Yong Li, and Shiyao Zhu. Effect of the casimir force on the entanglement between a levitated nanosphere and cavity modes. Phys. Rev. A 86(6), 063809 (2012).

[33] A. Arvanitaki and A. A. Geraci. Detecting HighFrequency Gravitational Waves with Optically Levitated Sensors. Phys. Rev. Lett. 110(7), 071105 (2013).

[34] R. Kaltenbaek, G. Hechenblaikner, N. Kiesel, O. RomeroIsart, K. C. Schwab, U. Johann, and M. Aspelmeyer. Macroscopic quantum resonators (MAQRO). Testing quantum and gravitational physics with massive mechanical resonators. Experimental Astronomy 34, 123-164 (2012).

[35] Y. Arita, A. W. McKinley, M. Mazilu, H. RubinszteinDunlop, and K. Dholakia. Picoliter rheology of gaseous media using a rotating optically trapped birefringent microparticle. Anal. Chem. 83(23), 8855 (2011).

[36] H. K. Cheung and C. K. Law, Optomechanical coupling between a moving dielectric sphere and radiation fields: A Lagrangian-Hamiltonian formalism. Phys. Rev. A 86, 033807 (2012).

[37] H. Shi and M. Bhattacharyaa, Coupling a small torsional oscillator to large optical angular momentum. Journal of Modern Optics 60(5), 382-386 (2013); H. Shi and M. Bhattacharya, Mechanical memory for photons with orbital angular momentum. J. Phys. B: At. Mol. Opt. Phys. 46(15), 151001 (2013).

[38] W. Lechner, S. J. M. Habraken, N. Kiesel, M. Aspelmeyer, P. Zoller, Phys. Rev. Lett. 110, 143604 (2013).

[39] S.J.M. Habraken, W. Lechner, P. Zoller, Phys. Rev. A 87, 053808 (2013).

[40] A. Ashkin and J. M. Dziedzic. Optical levitation by radiation pressure. Appl. Phys. Lett. 19, 283 (1971).

[41] A. Ashkin and J. M. Dziedzic. Optical levitation in high vacuum. Appl. Phys. Lett. 28, 333 (1976).

[42] Tongcang Li, Fundamental tests of physics with optically trapped microspheres, Springer, New York (2013).

[43] J. Gieseler, , L. Novotny, and R. Quidant. Thermal nonlinearities in a nanomechanical oscillator. arXiv e-prints 1307.4684, 2013.

[44] A. Einstein. Theoretische bemerkungen über die Brownsche bewegung. Zeit. f. Elektrochemie 13, 41 (1907).

[45] P. F. Cohadon, A. Heidmann, and M. Pinard. Cooling of a mirror by radiation pressure. Phys. Rev. Lett., 83(16), 3174-3177 (1999).

[46] O. Romero-Isart, L. Clemente, C. Navau, A. Sanchez, and J. I. Cirac, Quantum Magnetomechanics with Levitating Superconducting Microspheres. Phys. Rev. Lett. 109, 147205 (2012).

[47] M. Cirio, G. K. Brennen, and J. Twamley, Quantum Magnetomechanics: Ultrahigh-Q-Levitated Mechanical Oscillators. Phys. Rev. Lett. 109, 147206 (2012).

[48] T. A. Savard, K. M. O'Hara, and J. E. Thomas. Lasernoise-induced heating in far-off resonance optical traps. Phys. Rev. A 56(2), R1095-R1098 (1997).

[49] Thomas Corbitt and et al. Optical dilution and feedback 
cooling of a gram-scale oscillator to $6.9 \mathrm{mk}$. Phys. Rev. Lett. 99(16), 160801 (2007).

[50] Lajos Diósi. Laser linewidth hazard in optomechanical cooling. Phys. Rev. A 78(2), 021801 (2008).

[51] P. Rabl, C. Genes, K. Hammerer, and M. Aspelmeyer. Phase-noise induced limitations on cooling and coherent evolution in optomechanical systems. Phys. Rev. A 80(6), 063819 (2009).

[52] G. A. T. Pender, P. F. Barker, F. Marquardt, J. Millen, and T. S. Monteiro. Optomechanical cooling of levitated spheres with doubly resonant fields. Phys. Rev. A 85(2), 021802 (2012).

[53] Zhang-qi Yin and Y.-J. Han. Generating EPR beams in a cavity optomechanical system. Phys. Rev. A 79(2), 024301 (2009).

[54] Z.-q. Yin, T. Li, X. Zhang, and L. M. Duan. Raising Schrödinger's cat with a levitated nanodiamond through spin-opto-mechanical coupling. ArXiv e-prints 1305.1701, May 2013.

[55] V. R. Horowitz, B. J. Aleman, D. J. Christle, A. N. Cleland, and D. D. Awschalom. Electron spin resonance of nitrogen-vacancy centers in optically trapped nanodiamonds. Proceedings of the National Academy of Science 109, 13493-13497 (2012).

[56] M. Geiselmann, M. L. Juan, J. Renger, J. M. Say, L. J. Brown, F. J. G. de Abajo, F. Koppens, and R. Quidant. Three-dimensional optical manipulation of a single electron spin. Nature Nanotechnology 8, 175-179 (2013).

[57] L. P. Neukirch, J. Gieseler, R. Quidant, L. Novotny, and A. N. Vamivakas. Observation of nitrogen vacancy photoluminescence from an optically levitated nanodiamond. arXiv e-prints 1305.1515, May 2013.

[58] H. J. Mamin, M. Poggio, C. L. Degen, and D. Rugar. Nuclear magnetic resonance imaging with 90-nm resolution. Nature Nanotechnology 2, 301-306 (2007).

[59] P. Rabl, P. Cappellaro, M. V. Gurudev Dutt, L. Jiang, J. R. Maze, and M. D. Lukin. Strong magnetic coupling between an electronic spin qubit and a mechanical resonator. Phys. Rev. B 79(4), 041302 (2009).

[60] S. Kolkowitz, A. C. Bleszynski Jayich, Q. P. Unterreithmeier, S. D. Bennett, P. Rabl, J. G. E. Harris, and M. D. Lukin. Coherent Sensing of a Mechanical Resonator with a Single-Spin Qubit. Science 335, 1603-1606 (2012).

[61] L. Robledo, L. Childress, H. Bernien, B. Hensen, P. F. A. Alkemade, and R. Hanson. High-fidelity projective readout of a solid-state spin quantum register. Nature $\mathbf{4 7 7}$, 574-578 (2011).

[62] D. M. Meekhof, C. Monroe, B. E. King, W. M. Itano, and D. J. Wineland. Generation of nonclassical motional states of a trapped atom. Phys. Rev. Lett. 76(11), 17961799 (1996).

[63] G. Balasubramanian, P. Neumann, D. Twitchen, M. Markham, R. Kolesov, N. Mizuochi, J. Isoya, J. Achard, J. Beck, J. Tissler, V. Jacques, P. R. Hemmer, F. Jelezko, and J. Wrachtrup. Ultralong spin coherence time in isotopically engineered diamond. Nature Materials 8, 383-387 (2009).

[64] J. D. Thompson, B. M. Zwickl, A. M. Jayich, F. Marquardt, S. M. Girvin, and J. G. E. Harris. Strong dispersive coupling of a high-finesse cavity to a micromechanical membrane. Nature 452, 72-75 (2008).

[65] Xi Chen, A. Ruschhaupt, S. Schmidt, A. del Campo, D. Guéry-Odelin, and J. G. Muga. Fast optimal frictionless atom cooling in harmonic traps: Shortcut to adia- baticity. Phys. Rev. Lett. 104(6), 063002 (2010).

[66] H.J. Mamin and D. Rugar. Sub-attonewton force detection at millikelvin temperatures. Appl. Phys. Lett. 79, 3358 (2001).

[67] R. Maiwald et. al., Stylus ion trap for enhanced access and sensing. Nature Physics 5, 551-554 (2009); M. Biercuk et. al., Ultrasensitive detection of force and displacement using trapped ions. Nature Nanotechnology 5, 646$50(2010)$

[68] J.D.Teufel, T. Donner, M.A. Castellanos-Beltran, J.W. Harlow, K.W. Lehnert, Nanomechanical motion measured with an imprecision below that at the standard quantum limit. Nature Nanotech. 4, 820-823 (2009).

[69] D. Rugar et. al., Single spin detection by magnetic resonance force microscopy. Nature 430, 329-332 (2004).

[70] A. A. Geraci et. al., Improved constraints on nonNewtonian forces at 10 microns. Phys. Rev. D $\mathbf{7 8}(2)$, 022002 (2008).

[71] P. S. Epstein, On the Resistance Experienced by Spheres in their Motion through Gases. Phys. Rev. 23(6), 710-733 (1924).

[72] D.E. Chang et. al., Ultrahigh-Q mechanical oscillators through optical trapping. New. J. Phys. 14, 045002 (2012).

[73] Y. Hadjar et. al., High-sensitivity optical measurement of mechanical Brownian motion. Europhys. Lett. 47(5), 545-551 (1999).

[74] G. Anetsberger et. al., Near-field cavity optomechanics with nanomechanical oscillators. Nature Physics 5, 909914 (2009).

[75] E. G. Adelberger, J. H. Gundlach, B. R. Heckel, S. Hoedl, and S. Schlamminger, Torsion balance experiments: A low-energy frontier of particle physics. Prog. Part. Nucl. Phys 62, 102-134 (2009).

[76] A. O. Sushkov, W. J. Kim, D. A. R. Dalvit, and S. K. Lamoreaux, New Experimental Limits on NonNewtonian Forces in the Micrometer Range. Phys. Rev. Lett. 107(17), 171101 (2011).

[77] M. Masuda and M. Sasaki, Limits on Nonstandard Forces in the Submicrometer Range. Phys. Rev. Lett. 102(17), 171101 (2009).

[78] H. B. G. Casimir, On the attraction between two perfectly conducting plates. Proc. K. Ned. Akad. Wet. $\mathbf{5 1 , 7 9 3 ~ ( 1 9 4 8 ) . ~}$

[79] A.O. Sushkov et. al., Observation of the thermal Casimir force. Nature Physics 7, 230-233 (2011).

[80] R. S. Decca et. al., Tests of new physics from precise measurements of the Casimir pressure between two goldcoated plates. Phys. Rev. D 75, 077101 (2007).

[81] S. K. Lamoreaux, Demonstration of the Casimir Force in the 0.6 to $6 \mu \mathrm{m}$ Range. Phys. Rev. Lett. 78, 5 (1997).

[82] R. S. Decca et. al., Constraining New Forces in the Casimir Regime Using the Isoelectronic Technique. Phys. Rev. Lett. 94, 240401 (2005).

[83] A. Lambrecht and S. Reynuad, arXiv eprint 1112.1301v1 (2011).

[84] C.I.Sukenik et.al., Measurement of the Casimir-Polder force. Phys. Rev. Lett. 70, 560-563 (1993); D. M. Harber et. al., Measurement of the Casimir-Polder force through center-of-mass oscillations of a Bose-Einstein condensate. Phys. Rev. A 72, 033610 (2005).

[85] B.Abbott, et. al., LIGO: the Laser Interferometer Gravitational-Wave Observatory. Rep. Prog. Phys. 72, 076901 (2009). 
[86] G. M. Harry (for the LIGO Scientific Collaboration), Advanced LIGO: the next generation of gravitational wave detectors. Class. Quantum Grav. 27, 084006 (2010).

[87] T. Accadia et. al., Virgo: a laser interferometer to detect gravitational waves. Journal of Inst. 7, P030012 (2012); The Virgo Collaboration, note VIR-027A-09 (2009).

[88] H. Grote (for the LIGO Scientific Collaboration), The GEO 600 status. Class. Quantum Grav. 27084003 (2010); B. Willke et. al., The GEO-HF project. ibid. 23,
S207 (2006).

[89] K. Kuroda (for the LCGT Collaboration), Status of LCGT. Class. Quantum Grav. 27, 084004 (2010).

[90] A. Arvanitaki, S. Dimopoulos, S. Dubovsky, et. al., String axiverse. Phys. Rev. D 81, 123530 (2010); A. Arvanitaki and S. Dubovsky, Exploring the string axiverse with precision black hole physics. Phys. Rev. D 83, 044026 (2011). 